\title{
IRREGULAR MODIFIED $A$-HYPERGEOMETRIC SYSTEMS
}

\author{
FRANCISCO-JESÚS CASTRO-JIMÉNEZ, MARÍA-CRUZ FERNÁNDEZ-FERNÁNDEZ, \\ TATSUYA KOIKE, AND NOBUKI TAKAYAMA
}

\begin{abstract}
A modified $A$-hypergeometric system is a system of differential equations for the function $f\left(t^{w} \cdot x\right)$, where $f(y)$ is a solution of an $A$-hypergeometric system in $n$ variables and $w$ is an $n$-dimensional integer vector, which is called the weight vector. We study the irregularity of modified systems by adapting to this case the notion of umbrella introduced by M. Schulze and U. Walther. Especially, we study slopes and Gevrey series solutions. We develop some applications of this study. Under some conditions we give Laplace integral representations of divergent series solutions of the modified system and we show that certain Gevrey series solutions of the original $A$-hypergeometric system along coordinate varieties are Gevrey asymptotic expansions of holomorphic solutions of the $A$-hypergeometric system.
\end{abstract}

\section{INTRODUCTION}

A-Hypergeometric systems (or GKZ-systems or simply hypergeometric systems) are systems of linear partial differential equations on the complex affine space $\mathbb{C}^{n}$. Although they were already considered in works by J. Hrabowski [19] and by I. M. Gelfand, M. I. Graev and A. V. Zelevinsky [13, the systematic study of hypergeometric systems started with the paper by I. M. Gel'fand, M. M. Kapranov and A. V. Zelevinsky [14. Each of these systems, denoted by $H_{A}(\beta)$, is determined by a pair $(A, \beta)$, where $A=\left(a_{i j}\right)$ is an integer $d \times n$ matrix of rank $d$ and $\beta \in \mathbb{C}^{d}$ is a parameter vector. An $A$-hypergeometric system $H_{A}(\beta)$ is defined by the $d$ Euler operators $E_{i}-\beta_{i}:=\sum_{j=1}^{n} a_{i j} y_{j} \frac{\partial}{\partial y_{j}}-\beta_{i}$ for $i=1, \ldots, d$ and the toric operators $\partial^{u}-\partial^{v}$ associated with each pair $(u, v) \in \mathbb{N}^{n} \times \mathbb{N}^{n}$ such that $A u=A v$. Here $\partial^{u}$ stands for the monomial differential operator $\partial_{1}^{u_{1}} \cdots \partial_{n}^{u_{n}}$ and $\partial_{i}=\frac{\partial}{\partial y_{i}}$.

For generic parameters $\beta \in \mathbb{C}^{d}$, the holomorphic solutions of $H_{A}(\beta)$ at nonsingular points can be described by using the so-called $\Gamma$-hypergeometric series (see [13, [14; see also [26] and Subsection 2.2). We are interested in divergent $\Gamma$-hypergeometric series solutions. To study them we use the notion of slopes defined in the general setting by Y. Laurent 21]. If the matrix $A$ is pointed (which means that the column vectors of $A$ lie in an open half-space with boundary passing

Received by the editors September 9, 2012 and, in revised form, May 28, 2013.

2010 Mathematics Subject Classification. Primary 32C38, 14F10; Secondary 33C70, 13N10, $14 \mathrm{M} 25$.

The first and second authors were partially supported by MTM2010-19336 and FEDER and Junta de Andalucía FQM5849, FQM333.

The first author was also partially supported by S-13025-JSPS (Japan).

The second author was also partially supported by Max Planck Institute für Mathematik (Bonn).

The third author was partially supported by JSPS grants-in-aid No. 21740098 and No. S24224001. 
through the origin in $\left.\mathbb{R}^{d}\right)$, M. Schulze and U. Walther [27] have described the slopes of $H_{A}(\beta)$ with respect to coordinates varieties, generalizing previous work in [7], [16] and [17. These slopes are closely related to the irregularity of the system [22] and the existence of nonconvergent Gevrey series solutions of this system. Gevrey series solutions of $H_{A}(\beta)$ were studied by the second author in [10] (see also [1]. and 12 where the authors treat particular cases).

Modified hypergeometric systems were introduced by the fourth author [30] in order to study solutions of hypergeometric systems along a curve $y=\left(c_{1} t^{w_{1}}, \ldots, c_{n} t^{w_{n}}\right)$ for $w \in \mathbb{Z}^{n}, c_{i} \in \mathbb{C}$. Each of them is determined by a tuple $(A, w, \beta, \alpha)$, where $A$ and $\beta$ are as before, $w=\left(w_{1}, \ldots, w_{n}\right) \in \mathbb{Z}^{n}$ and $\alpha \in \mathbb{C}$. We denote by $\widetilde{A}(w)$ (or simply $\widetilde{A}$ ) the matrix

$$
\widetilde{A}(w)=\widetilde{A}=\left(\begin{array}{cccc}
a_{11} & \cdots & a_{1 n} & 0 \\
& \cdots & & 0 \\
a_{d n} & \cdots & a_{d n} & 0 \\
w_{1} & \cdots & w_{n} & 1
\end{array}\right) .
$$

Throughout this paper, we do not always assume that $A$ is pointed, but we assume that $\widetilde{A}$ is. Note that when $A$ is pointed, then $\widetilde{A}$ is as well.

Definition $0.1([30])$. We call the following system of differential equations $H_{A, w, \alpha}(\beta)$ a modified $A$-hypergeometric system:

$$
\begin{aligned}
\left(\sum_{j=1}^{n} a_{i j} x_{j} \partial_{j}-\beta_{i}\right) \bullet f & =0 \quad(i=1, \ldots, d), \\
\left(\sum_{j=1}^{n} w_{j} x_{j} \partial_{j}-t \partial_{t}-\alpha\right) \bullet f & =0, \\
\left(\prod_{i=1}^{n} \partial_{i}^{u_{i}} t^{u_{n+1}}-\prod_{j=1}^{n} \partial_{j}^{v_{j}} t^{v_{n+1}}\right) \bullet f & =0,
\end{aligned}
$$

with $u, v \in \mathbb{N}^{n+1}$ running over all $u, v$ such that $\widetilde{A} u=\widetilde{A} v$. Here we denote $\frac{\partial}{\partial x_{i}}=\partial_{i}$.

The modified system is defined on the space $X=\mathbb{C}^{n+1}$ with coordinates $(x, t)=$ $\left(x_{1}, \ldots, x_{n}, t\right)$. Let $D$ (or $\left.D_{n+1}\right)$ be the Weyl algebra in $(x, t)$. The left ideal in $D$ generated by the operators in (0.1), (0.2), and (0.3) is also denoted by $H_{A, w, \alpha}(\beta)$ if no confusion arises. The left $D$-module $D / H_{A, w, \alpha}(\beta)$ is denoted by $M_{A, w, \alpha}(\beta)$. By [30] the $D$-module $M_{A, w, \alpha}(\beta)$ is holonomic for any $A, \beta, w, \alpha$.

The system $H_{A}(\beta)$ is a summand of the modified system on the space $t \neq 0$. More precisely, denote $Y=\mathbb{C}^{n+1}$ with coordinates $(y, s)$ and consider the map

$$
\varphi: Y^{*}:=\mathbb{C}^{n} \times \mathbb{C}^{*} \subset Y \longrightarrow X^{*}:=\mathbb{C}^{n} \times \mathbb{C}^{*} \subset X
$$

defined by $\varphi\left(y_{1}, \ldots, y_{n}, s\right)=\left(s^{-w_{1}} y_{1}, \ldots, s^{-w_{n}} y_{n}, s\right)$. The pullback image of the ideal $H_{A, w, \alpha}(\beta)$ by $\varphi$ equals the ideal of differential operators on $Y^{*}$ generated by $H_{A}(\beta)$ and $s \partial_{s}+\alpha$. Notice that this last ideal is nothing but the hypergeometric ideal associated with the matrix $\widetilde{A}(\mathbf{0})$ and the parameter vector $(\beta,-\alpha) \in \mathbb{C}^{d+1}$. As usual we denote this ideal by $H_{\widetilde{A}(\mathbf{0})}(\beta,-\alpha)$.

Let us consider the local analytic situation. Let $\mathcal{D}$ be the sheaf of holomorphic differential operators on $X$ and $\mathcal{M}_{A, w, \alpha}(\beta)$ the quotient sheaf $\mathcal{D} / \mathcal{D} H_{A, w, \alpha}(\beta)$. 
By the previous observation, the hypergeometric $\mathcal{D}$-module $\mathcal{M}_{\widetilde{A}(\mathbf{0})}(\beta,-\alpha)=$ $\mathcal{D} / \mathcal{D} H_{\widetilde{A}(\mathbf{0})}(\beta,-\alpha)$ is the extension to $Y$ of the pullback module $\varphi^{*}\left(\mathcal{M}_{A, w, \alpha}(\beta)\right)$

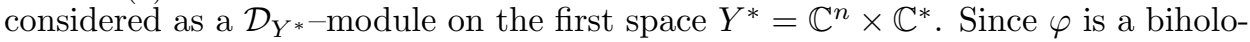
morphic map both $\mathcal{D}$-modules $\mathcal{M}_{\widetilde{A}(\mathbf{0})}(\beta,-\alpha)_{\mid Y^{*}}$ and $\mathcal{M}_{A, w, \alpha}(\beta)_{\mid X^{*}}$ are isomorphic.

We can describe solutions of the original hypergeometric system $H_{A}(\beta)$ associated to the weight vector $w \in \mathbb{Z}^{n}$ via solutions of the modified system. Series solutions of $H_{A}(\beta)$ have been studied in [13] and [14, where the authors constructed convergent series solutions associated to the regular triangulation obtained by a generic weight vector $w$. The construction is generalized as follows [26]: Assume that $\beta \in \mathbb{C}^{d}$ is very generic; this condition is essential in the construction. Suppose that the initial ideal $\operatorname{in}_{(-w, w)}\left(H_{A}(\beta)\right)$ has a solution of the form $y^{\rho}, \rho \in \mathbb{C}^{n}$. Then the monomial $y^{\rho}$ can be extended to a formal series solution $\phi(y)=y^{\rho}+\cdots$ of $H_{A}(\beta)$. We call the series $\phi(y)$ a series solution of $H_{A}(\beta)$ associated to the weight vector $w$. The series is divergent in general. We are interested in giving an explicit expression of a solution of $H_{A}(\beta)$ whose asymptotic expansion is $\phi(y)$. A standard method, in the theory of ordinary differential equations, to construct such an expression is the Laplace integral representation and the Borel transformation of divergent series. This method has been successful in the study of global analytic properties of solutions of ordinary differential equations; see, e.g., the book by W. Balser [3] and the references therein. Then it is a natural problem to construct a Laplace integral representation associated to the divergent series solution $\phi(y)$ associated to the weight vector $w$. Our modified system, which is a system of differential equations for $\phi\left(t^{w_{1}} x_{1}, \ldots, t^{w_{n}} x_{n}\right)$, is used to give an answer to this problem.

A key ingredient of our study is the fact that the modified hypergeometric system $H_{A, w, \alpha}(\beta)$ is transformed into the hypergeometric system $H_{\widetilde{A}(w)}(\beta, \alpha-1)$ associated with the matrix $\widetilde{A}(w)$ and parameter $(\beta, \alpha-1)$, by the formal inverse Fourier transformation $t \mapsto \partial_{t}, \partial_{t} \mapsto-t$, which enables us to study the modified system using the theory of hypergeometric systems (see Subsection 3.1).

For example, for $A=(1,2)$ and $\beta \in \mathbb{C}$ the system $H_{A}(\beta)$ is generated by $x_{1} \partial_{1}+2 x_{2} \partial_{2}-\beta$ and $\partial_{1}^{2}-\partial_{2}$. The modified system $H_{A, w, \alpha}(\beta)$ for $w=(-1,-1)$, $\alpha \in \mathbb{C}$ is generated by the three operators $x_{1} \partial_{1}+2 x_{2} \partial_{2}-\beta,-x_{1} \partial_{1}-x_{2} \partial_{2}-$ $t \partial_{t}-\alpha, \partial_{1}^{2} t-\partial_{2}$, and the inverse Fourier transformation of $H_{A, w, \alpha}(\beta)$ is generated by $x_{1} \partial_{1}+2 x_{2} \partial_{2}-\beta,-x_{1} \partial_{1}-x_{2} \partial_{2}+t \partial_{t}-\alpha+1, \partial_{1}^{2} \partial_{t}-\partial_{2}$, which is equal to $H_{\widetilde{A}(w)}(\beta, \alpha-1)$.

We study the behavior of solutions of $H_{A, w, \alpha}(\beta)$ near the hyperplane $t=0$ in the space $X$ and we give Laplace integral representations of its solutions.

The structure of the paper is as follows: in Section 1 we recall Y. Laurent's definition of the slopes of a finitely generated $D$-module with respect to a hypersurface.

In Section 2 we first recall the use of umbrellas for the description of the slopes of an $A$-hypergeometric system given by M. Schulze and U. Walther [27, and then we summarize the construction of the Gevrey solutions given in [10] and extend some of these results to the case of the Gevrey solutions at infinity.

In Section 3 we prove that the formal inverse Fourier transform, with respect to $T$, of a modified hypergeometric system is an $A$-hypergeometric system, and we use this fact to describe the slopes of the former by using the umbrella of the latter. We construct, associated with any slope of $\mathcal{M}_{A, w, \alpha}(\beta)$, a basis of its Gevrey solutions, modulo convergent power series, when the parameters $\beta$ and $\alpha$ are very generic; see 
Theorem 3.23. Moreover, for $\beta \in \mathbb{C}^{d}$ very generic we construct a basis of formal series solutions of the modified system for any $\alpha \in \mathbb{C}$ and $w \in \mathbb{Z}^{n}$; see Theorem 3.20. If in addition $w$ is generic, this basis is reduced to a single element. Later, in Sections 4and 5] we prove that this solution is an asymptotic expansion of a Laplace integral representation of a solution by the Borel summation method (Theorem 4.3 and Section 5). As an application, we give an asymptotic error evaluation of finite sums of formal series solutions of original $A$-hypergeometric systems with irregular singularities studied in, e.g., [10] and [14] (see Theorem 4.3, the inequality (4.7), and Section 5). Example 4.11 illustrates the application for the simplest $A$.

Several integral representations have been studied for solutions of regular holonomic $A$-hypergeometric systems (see, e.g., [15], 6], 4]). They play a prominent role in the study of $A$-hypergeometric functions. However, there have been few studies of integral representations for solutions of irregular $A$-hypergeometric systems. We would like to point out that integral representations of holomorphic solutions of irregular $A$-hypergeometric systems have been recently given by A. Esterov and K. Takeuchi [9] by using the so-called rapid decay homology cycles. Our Laplace integral representation, which we propose in this paper for giving an analytic meaning to divergent series solutions, is different from their representation: the integrand of our representation is an $A$-hypergeometric function associated to a homogenized configuration of $A$ (Section 4).

In Section 5 we illustrate how, under some conditions, the study of the irregularity of $\mathcal{M}_{A, w, \alpha}(\beta)$ along $T$ gives an analytic meaning to the Gevrey series solutions of $\mathcal{M}_{A}(\beta)$, along coordinate varieties, constructed in [10] by the second author. More precisely we prove (Remark 5.7 and Theorem [5.8) that they are asymptotic expansions of certain holomorphic solutions of $\mathcal{M}_{A}(\beta)$.

\section{Generalities on SlOPES}

Recall that $D=D_{n+1}$ is the Weyl algebra $\mathbb{C}\left\langle x_{1}, \ldots, x_{n}, t, \partial_{1}, \ldots, \partial_{n}, \partial_{t}\right\rangle$. In this section we write $x=\left(x_{1}, \ldots, x_{n+1}\right), \partial=\left(\partial_{1}, \ldots, \partial_{n+1}\right)$. The variable $t$ is also denoted by $x_{n+1}$ and $\partial_{t}$ by $\partial_{n+1}$.

Let $L: \mathbb{R}^{2 n+2} \rightarrow \mathbb{R}$ be a linear form $L(\alpha, \beta)=\sum_{i} u_{i} \alpha_{i}+v_{i} \beta_{i}$ such that $u_{i}+v_{i} \geq 0$ for $i=1, \ldots, n+1$, inducing the so-called $L$-filtration on the ring $D$. If $u_{i}+v_{i}>0$ for all $i$, the associated graded ring $\operatorname{gr}^{L}(D)$ is isomorphic to a polynomial ring in $2 n+2$ variables $(x, \xi)=\left(x, \xi_{1}, \ldots, \xi_{n+1}\right)$ with complex coefficients. This polynomial ring is $L$-graded, the $L$-degree of a monomial $x^{\alpha} \xi^{\beta}$ being $L(\alpha, \beta)$. If we need to emphasize the coefficients of the linear form we simply write $L=L_{(u, v)}$ for $(u, v) \in \mathbb{R}^{2 n+2}$ with $u_{i}+v_{i} \geq 0$ for all $i$. If $u=\mathbf{0} \in \mathbb{N}^{n+1}$ and $v=\mathbf{1}=(1,1, \ldots, 1) \in \mathbb{N}^{n+1}$, then the corresponding $L_{(u, v)}$ filtration is nothing but the usual order filtration on $D$ (which is also called the $F$-filtration). If $u=(\mathbf{0},-1) \in \mathbb{N}^{n+1}$ and $v=-u \in \mathbb{N}^{n+1}$, then the corresponding $L_{(u, v)}$ filtration is nothing but the Malgrange-Kashiwara filtration on $D$ (also known as the $V$-filtration) with respect to $t=0$. In the remainder of this section we assume $u_{i}+v_{i}>0$ for all $i$; we say then that $(u, v)$ is a weight vector for the Weyl algebra $D$.

All the $D$-modules appearing here are left $D$-modules unless stated otherwise. We denote by $T \subset \mathbb{C}^{n+1}$ the hyperplane defined by $t=0$.

Let $M$ be a finitely generated $D$-module. To the $L$-filtration on $D$ we associate a good $L$-filtration on $M$, by means of a finite presentation. The associated $\operatorname{gr}^{L}(D)$ module $\operatorname{gr}^{L}(M)$ is then finitely generated. The radical of the annihilating ideal 
$A n n_{\mathrm{gr}^{L}(D)}\left(\operatorname{gr}^{L}(M)\right)$, which is independent of the $\operatorname{good} L$-filtration on $M$, defines an affine algebraic subset of the cotangent space $T^{*} \mathbb{C}^{n+1}=\mathbb{C}^{2 n+2}$. This algebraic set is called the $L$-characteristic variety of $M$ and is denoted by $C h^{L}(M)$. The results stated so far are well known in $D$-module theory and generalize [5], which treats the case of the $F$-filtration in $D$. The case of a general $L$-filtration has been studied for example in [20] and, with more details, in [21, Section 3.2] in the microdifferential setting which is slightly different from the one in this paper. See also [2, Section 2] for an equivalent treatment better adapted to effective computations for modules on the Weyl algebra.

We consider a special type of $L$-filtration: For any real number $r \in \mathbb{R}_{\geq 0}$ we denote by $L_{r}$ either the linear form $L_{r}=F+r V$ or the filtration on $D$ given by the $(2 n+2)$-component weight vector $(0, \ldots, 0,-r, 1, \ldots, 1,1+r)$, where $-r$ is placed in the $(n+1)^{t h}$-component. Here $F$ (resp. $V$ ) stands for the order filtration on $D$ (resp. the Malgrange-Kashiwara filtration with respect to $T$ ).

Definition 1.1 ([21, Section 3.4]). Let $M$ be a finitely generated $D$-module. Consider the projection $\Pi: T^{*} \mathbb{C}^{n+1} \longrightarrow T$ defined by

$$
\Pi\left(x_{1}, \ldots, x_{n}, t, \xi_{1}, \ldots, \xi_{n}, \xi_{t}\right)=\left(x_{1}, \ldots, x_{n}, 0\right) .
$$

For any real number $r>0$, let $I_{T}^{r}(M)$ be the closure of the projection by $\Pi$ of the irreducible components of the $L_{r}$-characteristic variety $C h^{L_{r}} M \subset T^{*} \mathbb{C}^{n+1}$ that are not $(F, V)$-bihomogeneous. The real number $s=r+1>1$ is said to be a slope of $M$ along $T$ at $p \in T$ if and only if $p \in I_{T}^{r}(M)$.

As proved in [21, Section 3.4], any slope is a rational number and the set of slopes of $M$ is finite. Moreover, $s=r+1$ is a slope of $M$ along $T$ at $p \in T$ if and only if, in a neighborhood of $\Pi^{-1}(p), C h^{L_{r^{\prime}}}(M)$ is not locally constant for $r^{\prime} \in(r-\epsilon, r+\epsilon)$ with $\epsilon>0$ small enough; see loc. cit. We can define in a similar way the notion of slope with respect to any coordinate variety in $\mathbb{C}^{n+1}$.

\section{ON the IRREgularity OF $A$-HYPERGEOMETRIC Systems}

Recall that $A$ is a $d \times n$ integer matrix of rank $d$. We assume from now on that the column vectors of $A$ generate $\mathbb{Z}^{d}$ as a $\mathbb{Z}$-module. Recall that $H_{A}(\beta)$ is the hypergeometric ideal associated with $A$ and the parameter vector $\beta \in \mathbb{C}^{d}$. We denote by $M_{A}(\beta)$ the corresponding hypergeometric system (i.e., the quotient of $D_{n}:=\mathbb{C}\left\langle x_{1}, \ldots, x_{n}, \partial_{1}, \ldots, \partial_{n}\right\rangle$, the Weyl algebra of order $n$, by its left ideal $\left.H_{A}(\beta)\right)$. In this section we denote $X=\mathbb{C}^{n}$.

2.1. Slopes of $A$-hypergeometric systems. We recall in this subsection some results from [27], where $A$ is assumed to be pointed. These results will not be applied to our matrix $A$ but only to the matrix $\widetilde{A}(w)$ (see Subsection 3.2), which we assume to be pointed throughout this article.

We denote by $a_{i}$ the $i$-th column of $A$ for $i=1, \ldots, n$. The $L$-characteristic variety of the hypergeometric system $M_{A}(\beta)$ has been described, in a combinatorial way, by M. Schulze and U. Walther [27] for any pointed matrix $A$ and any filtration $L=(u, v)$ such that $u_{i}+v_{i}=c>0$ for all $i=1, \ldots, n$. The case $L=F$ was first studied by A. Adolphson [1]. The main tool for their description is the notion of an $(A, L)$-umbrella that we define here for the sake of completeness. First of all, the $(A, L)$-umbrella only depends on $A$ and on the coefficients $v_{i}$ of the linear form $L=L_{(u, v)}$. 
Definition 2.1 ([27, Def. 2.7]). We assume that $v_{i}>0$ for all $i$. The $(A, L)-$ polyhedron $\Delta_{A}^{L}$ is the convex hull in $\mathbb{R}^{d}$ of the set $\left\{\mathbf{0}, a_{1} / v_{1}, \ldots, a_{n} / v_{n}\right\}$. The $(A, L)$-umbrella $\Phi_{A}^{L}$ is the set of faces of $\Delta_{A}^{L}$ which do not contain zero. In particular, $\Phi_{A}^{L}$ contains the empty face.

By $\Phi_{A}^{L, k} \subset \Phi_{A}^{L}$ we denote the subset of faces of dimension $k$. We identify each face $\sigma$ of $\Phi_{A}^{L}$ with the set $\left\{i: a_{i} / v_{i} \in \sigma\right\}$ and with $\left\{a_{i}: a_{i} / v_{i} \in \sigma\right\}$. The $(A, L)$-umbrella is then an abstract cell complex.

When not all the $v_{i}$ are strictly positive, then both definitions of the $(A, L)$ polyhedron and the $(A, L)$-umbrella are a little bit more involved. We refer to [27. Def. 2.7] for these definitions in the general case. See also Subsection 3.2

Corollary 4.17 in 27] gives a description of the $L$-characteristic variety of $M_{A}(\beta)$ in terms of the $(A, L)$-umbrella. As a consequence we have a combinatorial description of the slopes of $M_{A}(\beta)$ at the origin along any coordinate variety $Y \subset X$ via considering the 1-parameter family of filtrations $L_{r}=F+r V, r>0$, where $V$ is the $V$-filtration 1 along $Y$ :

Theorem 2.2 ([27, Cor. 4.18]). The real number $s=r+1>1$ is a slope of $M_{A}(\beta)$ along $Y$ at the origin if and only if $\Phi_{A}^{L_{r^{\prime}}}$ is not locally constant at $r^{\prime}=r$.

Remark 2.3. When $Y \subset X$ is a coordinate hyperplane, then the set of slopes of $M_{A}(\beta)$ along $Y$ at the origin coincides with the set of slopes of $M_{A}(\beta)$ along $Y$ at any point $p \in Y$. This is proved in [10, Th. 5.9] by using the comparison result in [22, Th. 2.4.2].

2.2. Gevrey solutions of $A$-hypergeometric systems at infinity. We extend some of the results from [10] to the case of the Gevrey solutions of a hypergeometric system at infinity in the direction of a coordinate hyperplane that we may assume to be $x_{n}=0$. This construction is used later in the study of the Gevrey solutions along $T$ of a modified hypergeometric system; see Subsection 3.5 ,

Let us denote by $\mathcal{O}_{X}$ the sheaf of holomorphic functions on $X=\mathbb{C}^{n}$. For $Y=\left\{x_{n}=0\right\}$, we denote by $\mathcal{O}_{\widehat{X \mid Y}}$ the formal completion of $\mathcal{O}_{X}$ along $Y$, whose germs at $(p, 0) \in Y$ are of the form $f=\sum_{m \geq 0} f_{m} x_{n}^{m}$, where all the $f_{m}=f_{m}\left(x_{1}, \ldots, x_{n-1}\right)$ are holomorphic functions in a common neighborhood of $p$. Notice that the restriction of $\mathcal{O}_{X}$ to $Y$, denoted by $\mathcal{O}_{X \mid Y}$, is a subsheaf of $\mathcal{O}_{\widehat{X \mid Y}}$. For any real number $s$, we also consider the sheaf $\mathcal{O}_{\widehat{X \mid Y}}(s)$ of the Gevrey series along $Y$ of order $s$ which is defined to be the subsheaf of $\mathcal{O}_{\widehat{X \mid Y}}$ whose germs $f$ at $(p, 0) \in Y$ satisfy

$$
\sum_{m \geq 0} \frac{f_{m}}{m !^{s-1}} x_{n}^{m} \in \mathcal{O}_{X \mid Y,(p, 0)} .
$$

We denote $\mathcal{Q}_{Y}(s):=\frac{\mathcal{O}_{\widehat{X \mid Y}}(s)}{\mathcal{O}_{X \mid Y}}$ and use $\mathcal{O}_{\widehat{X \mid Y}}(<s)$ for the sheaf of the Gevrey series along $Y$ of order less than $s$. If $f$ belongs to $\mathcal{O}_{\widehat{X \mid Y}}(s) \backslash \mathcal{O}_{\widehat{X \mid Y}}(<s)$ for some $s$, we say that the index of the Gevrey series $f$ is $s$. We also write $\mathcal{O}_{\widehat{X \mid Y}}(+\infty):=\mathcal{O}_{\widehat{X \mid Y}}$.

We denote by $\mathcal{D}=\mathcal{D}_{X}$ the sheaf of linear differential operators on $X$ with holomorphic coefficients. If $\mathcal{M}$ is a coherent $\mathcal{D}$-module, Z. Mebkhout has defined

\footnotetext{
${ }^{1}$ The $V$-filtration with respect to the coordinate variety $Y=\left(x_{1}=\cdots=x_{\ell}=0\right)$ is defined by assigning the weight -1 (resp. the weight 1 ) to the variables $x_{i}\left(\operatorname{resp} . \partial_{i}\right)$ for $i=1, \ldots, \ell$ and the weight 0 to the remaining variables.
} 
in [24, Déf. 6.3.1] the irregularity of order $s$ of $\mathcal{M}$ with respect to $Y$ to be the complex of (sheaves of) vector spaces $\operatorname{Irr}_{Y}^{(s)}(\mathcal{M}):=\mathbb{R} \mathcal{H o m}_{\mathcal{D}}\left(\mathcal{M}, \mathcal{Q}_{Y}(s)\right)$ and has proved that, for all $s \in[1,+\infty]$, this complex is a perverse sheaf on $Y$ when $\mathcal{M}$ is holonomic [24, Th. 6.3.3]. By the comparison theorem [22, Th.2.4.2] $s$ is a slope of $\mathcal{M}$ with respect to $Y$ if and only if $s$ is a gap in the filtration $\operatorname{Irr}_{Y}^{(s)}(\mathcal{M})$ on the irregularity $\operatorname{Irr}_{Y}(\mathcal{M}):=\operatorname{Irr}_{Y}^{(+\infty)}(\mathcal{M})$. The perversity result implies, in particular, that at a generic point $p \in Y$ only the first cohomology space of previous complexes is possibly nonzero, and thus it is worth studying the stalk $\mathcal{H o m}_{\mathcal{D}}\left(\mathcal{M}, \mathcal{Q}_{Y}(s)\right)_{p}$.

Recall that $A=\left(a_{1} \cdots a_{n}\right)$ is a full rank matrix with $a_{i} \in \mathbb{Z}^{d}$ for all $i=1, \ldots, n$ and $d \leq n$. Following [14] and [26], for any vector $v \in \mathbb{C}^{n}$ we can define a series

$$
\phi_{v}=\phi_{v}(x):=\sum_{u \in N_{v}} \frac{[v]_{u_{-}}}{[v+u]_{u_{+}}} x^{v+u}
$$

where $v \in \mathbb{C}^{n}$ verifies $A v=\beta$ and $N_{v}=\left\{u \in \operatorname{ker}(A) \cap \mathbb{Z}^{n}: \operatorname{nsupp}(v+u)=\right.$ $\operatorname{nsupp}(v)\}$. Here $\operatorname{ker}(A)=\left\{u \in \mathbb{Q}^{n}: A u=0\right\}, \operatorname{nsupp}(w):=\left\{i \in\{1, \ldots, n\}: w_{i} \in\right.$ $\left.\mathbb{Z}_{<0}\right\}$ is the negative support of $w \in \mathbb{C}^{n},[v]_{u}=\prod_{i}\left[v_{i}\right]_{u_{i}}$ and $\left[v_{i}\right]_{u_{i}}=\prod_{j=1}^{u_{i}}\left(v_{i}-j+1\right)$ is the Pochhammer symbol for $v_{i} \in \mathbb{C}, u_{i} \in \mathbb{N}$.

The series $\phi_{v}$ is annihilated by the hypergeometric ideal $H_{A}(\beta)$ if and only if the negative support of $v$ is minimal, i.e., $\nexists u \in \operatorname{ker}(A) \cap \mathbb{Z}^{n}$ with $\operatorname{nsupp}(v+u) \subsetneq \operatorname{nsupp}(v)$ (see [26, Section 3.4]).

When $\beta \in \mathbb{C}^{d}$ is very generic, i.e., when $\beta$ is not in a locally finite countable union of Zarisky closed sets, there is a basis of the Gevrey solution space of $\mathcal{M}_{A}(\beta)$ along $Y$ given by series $\phi_{v}$ for suitable vectors $v \in \mathbb{C}^{n}$ (see [10, Ths. 6.2 and 6.7]).

For any subset $\eta \subseteq\{1, \ldots, n\}$ we denote by $A_{\eta}$ the submatrix of $A$ given by the columns of $A$ indexed by $\eta$ and we denote $\bar{\eta}=\{1, \ldots, n\} \backslash \eta$.

We say that $\sigma \subseteq\{1, \ldots, n\}$ is a $(d-1)$-simplex with respect to $A$ (or simply that $\sigma$ is a $(d-1)$-simplex) if the columns of $A_{\sigma}$ determine a basis of $\mathbb{R}^{d}$. If it is so, we can reorder the variables in order to have $\sigma=\{1, \ldots, d\}$ without loss of generality. Then a basis of $\operatorname{ker}(A)$ associated with $\sigma$ is given by the columns of the matrix

$$
B_{\sigma}=\left(\begin{array}{cccc}
-A_{\sigma}^{-1} a_{d+1} & -A_{\sigma}^{-1} a_{d+2} & \cdots & -A_{\sigma}^{-1} a_{n} \\
1 & 0 & & 0 \\
0 & 1 & & 0 \\
\vdots & & \ddots & \vdots \\
0 & 0 & & 1
\end{array}\right) .
$$

A vector $v \in \mathbb{C}^{n}$ satisfying $v_{i} \in \mathbb{N}$ for all $i \notin \sigma$ and $A v=\beta$ can be written as

$$
v^{\mathbf{k}}=\left(A_{\sigma}^{-1}\left(\beta-\sum_{i \notin \sigma} k_{i} a_{i}\right), \mathbf{k}\right)
$$

for some $\mathbf{k}=\left(k_{i}\right)_{i \notin \sigma} \in \mathbb{N}^{n-d}$. Since $\beta$ is very generic, then the negative support of $v^{\mathbf{k}}$ is the empty set and hence $\phi_{v^{\mathbf{k}}}$ is annihilated by $H_{A}(\beta)$. Moreover, the summation index set $N_{v^{\mathbf{k}}}$ in the series $\phi_{v^{\mathbf{k}}}$ is given by the integer vectors in an affine translate of the positive span of the columns of $B_{\sigma}$. The sum of the coordinates of the $i$-th column of $B_{\sigma}$ is $1-\left|A_{\sigma}^{-1} a_{d+i}\right|$, where || means the sum of the coordinates.

By Theorem 2.2, a real number $s>1$ is a slope of $M_{A}(\beta)$ along $Y=\left\{x_{n}=0\right\}$ if and only if $\frac{1}{s} a_{n}$ belongs to the hyperplane $H_{\tau}$ supported on a facet $\tau$ of the convex hull of $\left\{0, a_{1}, \ldots, a_{n-1}\right\}$ such that $0 \notin \tau$. In particular, for any $(d-1)$-simplex 
$\sigma \subseteq \tau$ it is easy to check that $s=\left|A_{\sigma}^{-1} a_{n}\right|>1$. We say in this case that $\sigma$ is a $(d-1)$-simplex corresponding to the slope $s>1$ of $M_{A}(\beta)$ along $Y$.

The following theorem is a summary of some of the results from [10. Its last statement uses results from [22] and in 27]. For the definition of a regular triangulation, see, e.g., [29, Ch. 8].

Theorem 2.4. Assume that $\beta \in \mathbb{C}^{d}$ is very generic and that $s>1$ is a slope of $M_{A}(\beta)$ along $Y=\left\{x_{n}=0\right\}$. For any $(d-1)$-simplex $\sigma$ corresponding to $s$, one can construct $\operatorname{vol}(\sigma)=\left|\operatorname{det}\left(A_{\sigma}\right)\right|$ many linearly independent Gevrey solutions $\phi_{v^{\mathbf{k}}}$ of $M_{A}(\beta)$ along $Y$ with index $s$ by varying $\mathbf{k} \in \mathbb{N}^{n-d}$ in a set $\Lambda$ so that $\left\{A_{\bar{\sigma}} \mathbf{k} \mid \mathbf{k} \in \Lambda\right\}$ is a set of representatives of the group $\mathbb{Z}^{d} / \mathbb{Z} A_{\sigma}$. Moreover, if we repeat this construction for all the $(d-1)$-simplices $\sigma$ corresponding to $s$ which belong to a suitable regular triangulation for $A$ and take the classes modulo $\mathcal{O}_{\widehat{X \mid Y}}(<s)$, we obtain a basis for the space of solutions of $M_{A}(\beta)$ in the space $\left(\mathcal{O}_{\widehat{X \mid Y}}(s) / \mathcal{O}_{\widehat{X \mid Y}}(<s)\right)_{p}$ for points $p \in Y$ in a relatively open set of $Y$.

We have exhibited the construction of the Gevrey solutions of $M_{A}(\beta)$ along $Y=\left\{x_{n}=0\right\}$ corresponding to each slope $s>1$ of $M_{A}(\beta)$ along $Y$ for $\beta$ very generic.

Let us construct Gevrey solutions of $M_{A}(\beta)$ at infinity. In other words, we are going to construct Gevrey solutions of the projectivized hypergeometric system treated in [28, Section 5] at a generic point at infinity. We use the following notation: $X^{\prime}$ is $\mathbb{C}^{n}$ with coordinates $\left(x_{1}, \ldots, x_{n-1}, z\right)$ and $z=1 / x_{n}$ so that $X \cap X^{\prime}=\mathbb{C}^{n-1} \times$ $\mathbb{C}^{*}$. Denote $Y^{\prime}=\left\{x_{n}=\infty\right\}=\{z=0\} \subseteq X^{\prime}$.

Take $L_{-r}=F-r V$, where $V$ is the $V$-filtration along $Y$. Notice that $\Phi_{A}^{L_{-r}}$ is not locally constant at $r=s-1>0$ if and only if $\frac{1}{(2-s)} a_{n}$ belongs to the hyperplane $H_{\tau}$ supported on a facet $\tau$ of the convex hull of $\left\{0, a_{1}, \ldots, a_{n-1}\right\}$ such that $0 \notin \tau$.

Theorem 2.5. Assume that $\beta \in \mathbb{C}^{d}$ is very generic and that there exists $s>1$ such that $\frac{1}{(2-s)} a_{n}$ belongs to a hyperplane $H_{\tau}$ as above. For any $(d-1)$-simplex $\sigma \subseteq \tau$ one can construct $\operatorname{vol}(\sigma)=\left|\operatorname{det}\left(A_{\sigma}\right)\right|$ many linearly independent Gevrey series $\phi_{v^{\mathbf{k}}}$ along $Y^{\prime}$ with index s by varying $\mathbf{k} \in \mathbb{N}^{n-d-1} \times \mathbb{Z}_{<0}$ in a set $\Lambda$ so that $\left\{A_{\bar{\sigma}} \mathbf{k} \mid \mathbf{k} \in \Lambda\right\}$ is a set of representatives of the group $\mathbb{Z}^{d} / \mathbb{Z} A_{\sigma}$. The classes of these series modulo convergent series $\mathcal{O}_{X^{\prime} \mid Y^{\prime}}$ are solutions of $M_{A}(\beta)$ in $\mathcal{O}_{\widehat{X^{\prime} \mid Y^{\prime}}}(s) / \mathcal{O}_{X^{\prime} \mid Y^{\prime}}$.

Moreover, if we repeat this construction for all the $(d-1)$-simplices $\sigma$ as above which belong to a suitable regular triangulation for $A$ and take the classes modulo $\mathcal{O}_{\widehat{X^{\prime} \mid Y^{\prime}}}(<s)$, then we obtain a basis for the space of solutions of $M_{A}(\beta)$ in the space $\left(\mathcal{O}_{\widehat{X^{\prime} \mid Y^{\prime}}}(s) / \mathcal{O}_{\widehat{X^{\prime} \mid Y^{\prime}}}(<s)\right)_{p}$ for points $p \in Y^{\prime}$ in a relatively open set of $Y^{\prime}$.

Proof. By reordering the variables we may assume $\sigma=\{1, \ldots, d\}$ without loss of generality. Let $B_{\sigma}^{\prime}$ be the matrix $B_{\sigma}$ but with the last column multiplied by -1 .

Take a vector $v \in \mathbb{C}^{n}$ such that $A v=\beta, v_{i} \in \mathbb{N}$ for all $i \notin \sigma \cup\{n\}$ and $v_{n} \in \mathbb{Z}_{<0}$. It is clear that such a vector can be taken as $v=v^{\mathbf{k}}$, as before, for some $\mathbf{k}=\left(k_{i}\right)_{i \notin \sigma} \in \mathbb{N}^{n-d-1} \times \mathbb{Z}_{<0}$. Moreover, the summation index set $N_{v^{\mathbf{k}}}$ in the series $\phi_{v^{\mathbf{k}}}$ is given by the integer vectors in an affine translate of the positive span of the columns of $B_{\sigma}^{\prime}$. Notice that the sum of the coordinates of the $i$-th column of $B_{\sigma}^{\prime}$ is $1-\left|A_{\sigma}^{-1} a_{d+i}\right| \geq 0$ for $i=1, \ldots, n-d-1$, while the sum of the coordinates of the last column is $\left|A_{\sigma}^{-1} a_{n}\right|-1=2-s-1=1-s<0$. This implies that $\phi_{v^{\mathbf{k}}}^{\prime}\left(x_{1}, \ldots, x_{n-1}, z\right):=\phi_{v^{\mathbf{k}}}\left(x_{1}, \ldots, x_{n-1}, 1 / z\right)$ is a Gevrey series along $Y^{\prime}$ with 
order $s=r+1>1$. To prove that $s$ is the Gevrey index of $\phi_{v^{\mathbf{k}}}^{\prime}$ one can use a slightly modified version of Lemma 3.8 in 10 .

Moreover, since $\beta$ is very generic, then the negative support of $v^{\mathbf{k}}$ is nsupp $\left(v^{\mathbf{k}}\right)=$ $\{n\}$ which is not minimal, and hence $\phi_{v^{\mathbf{k}}}$ is not annihilated by $H_{A}(\beta)$ (see 26 , Section 3.4]). However, it can be checked that for any differential operator $P \in$ $H_{A}(\beta)$ the series $P\left(\phi_{v^{\mathbf{k}}}\right)$ is either zero or a polynomial in $z=x_{n}^{-1}$ with coefficients that are convergent power series in the variables $x_{1}, \ldots, x_{n-1}$. Now we finish the proof following [10, Sec. 7] and [28, Sec. 5].

Remark 2.6. A slightly weaker version of the first paragraph of Theorem 2.5] can also be proved without the genericity assumption in $\beta \in \mathbb{C}^{d}$. More precisely, for all $\beta \in \mathbb{C}^{d}$, if $\Phi_{A}^{L_{-r}}$ is not locally constant at $r=s-1>0$ we can construct a Gevrey series along $Y^{\prime}$ of index $s=r+1$ which is a solution of $M_{A}(\beta)$ modulo the space $\mathcal{O}_{\widehat{X^{\prime} \mid Y^{\prime}}}(<s)$ (of Gevrey series along $Y^{\prime}$ with order less than $s$ ) by methods similar to the ones in [10, Section 4].

The following corollary is a particular case of [28, Conjecture 5.18].

Corollary 2.7. The real number $s>1$ is a slope of $M_{A}(\beta)$ along $Y^{\prime}=\left\{x_{n}=\infty\right\}$ if and only if $\Phi_{A}^{L_{-r}}$ is not locally constant at $r=s-1$.

Proof. For the only if direction of the proof we refer to [28, Section 5]. Let us prove the if direction. By Theorem 2.5 and Remark 2.6 one can construct a Gevrey series of index $s=r+1$ that is a solution of $M_{A}(\beta)$ along $Y^{\prime}\left(\operatorname{modulo} \mathcal{O}_{\widehat{X^{\prime} \mid Y^{\prime}}}(<s)\right)$. So, the result follows from the comparison theorem for the slopes [22].

Example 2.8. Set

$$
A=\left(\begin{array}{rrr}
2 & 1 & 1 \\
1 & 2 & 1
\end{array}\right)
$$

and $\beta \in \mathbb{C}^{2}$. We have that the $\operatorname{ker}(A)$ is generated by $u=(1,1,-3)$, and thus the hypergeometric ideal $H_{A}(\beta)$ is generated by $\square_{u}=\partial_{1} \partial_{2}-\partial_{3}^{3}, E_{1}-\beta_{1}=2 x_{1} \partial_{1}+$ $x_{2} \partial_{2}+x_{3} \partial_{3}-\beta_{1}$ and $E_{2}-\beta_{2}=x_{1} \partial_{1}+2 x_{2} \partial_{2}+x_{3} \partial_{3}-\beta_{2}$. Take $\sigma=\{1,2\}$ and notice that for $s=4 / 3$ we have that $a_{3} /(2-s)$ belongs to the line $H_{\sigma}$ determined by $a_{1}$ and $a_{2}$. By Corollary 2.7 we have that $s=4 / 3$ is a slope of $M_{A}(\beta)$ along $Y^{\prime}=\left\{x_{3}=\infty\right\}$. Indeed, if we consider $v^{\mathbf{k}}=\left(\left(2 \beta_{1}-\beta_{2}-k\right) / 3,\left(2 \beta_{2}-\beta_{1}-k\right) / 3, k\right)$ for $\mathbf{k}=k \in \mathbb{Z}_{<0}$ we have that $\phi_{v^{\mathbf{k}}}$ is a Gevrey series along $Y^{\prime}$ of index $s=4 / 3$ if $v_{1}^{\mathbf{k}}, v_{2}^{\mathbf{k}} \notin \mathbb{Z}_{<0}$. Moreover, we have that $\left(E_{i}-\beta_{i}\right)\left(\phi_{v^{\mathbf{k}}}\right)=0$ for $i=1,2$. For each of the three biggest $k \in \mathbb{Z}_{<0}$ verifying that $v_{1}^{k}, v_{2}^{k} \notin \mathbb{Z}_{<0}$ we have that $\square_{u}\left(\phi_{v^{k}}\right)=v_{1}^{k} v_{2}^{k} x_{1}^{v_{1}^{k}-1} x_{2}^{v_{2}^{k}-1} x_{3}^{k}$, which is convergent at any $p \in Y^{\prime} \cap\left\{x_{1} x_{2} \neq 0\right\}$. The classes modulo $\mathcal{O}_{X^{\prime} \mid Y^{\prime}}$ of these three series $\phi_{v^{\mathbf{k}}}$ form a basis for the space of solutions of $M_{A}(\beta)$ in $\left(\mathcal{O}_{\widehat{X^{\prime} \mid Y^{\prime}}}(s) / \mathcal{O}_{X^{\prime} \mid Y^{\prime}}\right)_{p}$, $p \in Y^{\prime} \cap\left\{x_{1} x_{2} \neq 0\right\}$. If $\beta$ is very generic, then $k=-1,-2,-3$.

\section{On the IRREgUlarity OF MODIFIED $A$-HYPERGEOMETRIC SYSTEMS}

3.1. Fourier transform and initial ideals. We define the ring isomorphism $\mathcal{F}$ of $D=D_{n+1}$ by $t \mapsto-\partial_{t}, \partial_{t} \mapsto t$. The isomorphism $\mathcal{F}$ is called the Fourier transform on $D$ with respect to the variable $t$. The inverse transform $\mathcal{F}^{-1}$ is given by $t \mapsto \partial_{t}$, $\partial_{t} \mapsto-t$. Let $(u, v)$ be a weight vector for the Weyl algebra. The Fourier transforms $\mathcal{F}$ and $\mathcal{F}^{-1}$ induce isomorphisms in $\operatorname{gr}^{L}(D)$, and we denote them also by $\mathcal{F}$ and $\mathcal{F}^{-1}$ respectively. Analogously, if $C \subset \mathbb{C}^{2 n+2}$ is the affine algebraic set defined 
by an ideal $J \subseteq \operatorname{gr}^{L}(D)$, we write $\mathcal{F} C$ and $\mathcal{F}^{-1} C$ for the algebraic set defined by $\mathcal{F} J \subseteq \operatorname{gr}^{L}(D)$ and $\mathcal{F}^{-1} J \subseteq \operatorname{gr}^{L}(D)$ respectively.

We define the Fourier transform of the weight vector $(u, v)$ by the formula $\mathcal{F}(u, v):=\left(u_{1}, \ldots, u_{n}, v_{n+1}, v_{1}, \ldots, v_{n}, u_{n+1}\right)$. We notice that $\mathcal{F} \mathcal{F}(u, v)=(u, v)$. We also write $\mathcal{F} L=\mathcal{F}(u, v)$ if $L=L_{(u, v)}$.

We have the following simple, but important, claim for the Gröbner deformation method.

Proposition 3.1. For any left ideal $I$ in $D$, we have

$$
\operatorname{in}_{(u, v)}(I)=\mathcal{F}^{-1} \operatorname{in}_{\mathcal{F}(u, v)}(\mathcal{F} I) .
$$

3.2. Slopes of modified $A$-hypergeometric systems. We retain the notation of [30, which are explained in the introduction. We are interested in the slopes of the modified system $M_{A, w, \alpha}(\beta)$ at any point along $T=\{t=0\}$. We notice that $H_{A, w, \alpha}(\beta)$ is the Fourier transform of $H_{\widetilde{A}(w)}(\tilde{\beta})$ where $\tilde{\beta}=(\beta, \alpha-1)$, i.e.,

$$
M_{A, w, \alpha}(\beta)=\mathcal{F} M_{\widetilde{A}(w)}(\widetilde{\beta}) .
$$

Recall that $L_{r}=F+r V=(0, \ldots, 0,-r, 1, \ldots, 1,1+r)$, where $F=(\mathbf{0}, \mathbf{1})$ and $V=(0, \ldots, 0,-1 ; 0, \ldots, 0,1)$. Thus $\mathcal{F} L_{r}=(0, \ldots, 0,1+r, 1, \ldots, 1,-r)$.

From Proposition 3.1 and (3.1), we have

$$
C h^{L_{r}}\left(M_{A, w, \alpha}(\beta)\right)=\mathcal{F}^{-1}\left(C h^{\mathcal{F} L_{r}}\left(M_{\widetilde{A}(w)}(\widetilde{\beta})\right)\right) .
$$

On the other hand, since $M_{\widetilde{A}(w)}(\widetilde{\beta})$ is a hypergeometric system and the matrix $\widetilde{A}(w)$ is pointed, Corollary 4.17 of [27] gives a description of the irreducible components of $C h^{\mathcal{F} L_{r}}\left(M_{\widetilde{A}(w)}(\widetilde{\beta})\right)$ in terms of the $\left(\widetilde{A}(w), \mathcal{F} L_{r}\right)$-umbrella. We notice here that the last coordinate of $\mathcal{F} L_{r}$ equals $-r<0$. We recall the definition [27, Def. 2.7] of the umbrella in this case. First of all let us recall loc. cit., that if $a, b$ are two points and $H$ is a hyperplane in $\mathbb{P}^{d+1}(\mathbb{R})$ containing neither $a$ nor $b$, then the convex hull $\operatorname{conv}_{H}(a, b)$ of $a$ and $b$ relative to $H$ is the unique line segment joining $a$ and $b$ and not meeting $H$.

Let us denote the $i$-th column of $\widetilde{A}(w)$ by $\widetilde{a}_{i}$, say $\widetilde{a}_{i}=\left(\begin{array}{c}a_{i} \\ w_{i}\end{array}\right)$ for $i=1, \ldots, n$ and $\widetilde{a}_{n+1}=(0, \ldots, 0,1)^{t}$. For simplicity let us write $(\widetilde{A}, \widetilde{L})$ instead of $\left(\widetilde{A}(w), \mathcal{F} L_{r}\right)$. We view $\widetilde{a}_{1}, \ldots, \widetilde{a}_{n+1}$ as points in $\mathbb{R}^{d+1} \subset \mathbb{P}^{d+1}(\mathbb{R})$. As $\widetilde{A}$ is pointed, there exists a linear form $h$ on $\mathbb{R}^{d+1}$ such that $h\left(\widetilde{a}_{i}\right)>0$ for all $i$. Let $\epsilon \in \mathbb{R}$ be such that $0<\epsilon<h\left(\widetilde{a}_{i}\right)$ for $i=1, \ldots, n$ and $0<\epsilon<\frac{h\left(\widetilde{a}_{n+1}\right)}{r}$.

Definition 3.2 ([27, Def. 2.7]). The $(\widetilde{A}, \widetilde{L})$-polyhedron $\Delta_{\widetilde{A}}^{\widetilde{L}}$ is the convex hull

$$
\Delta_{\widetilde{A}}^{\widetilde{L}}=\operatorname{conv}_{\mathrm{H}_{\epsilon}}\left(\left\{\mathbf{0}, \widetilde{a}_{1}, \ldots, \widetilde{a}_{n}, \frac{\widetilde{a}_{n+1}}{-r}\right\}\right) \subset \mathbb{P}^{d+1}(\mathbb{R}),
$$

where $H_{\epsilon}$ is the projective closure of the affine hyperplane $h^{-1}(-\epsilon)$. The $(\widetilde{A}, \widetilde{L})$ umbrella $\Phi_{\widetilde{A}}^{\widetilde{L}}$ is the set of faces of $\Delta_{\widetilde{A}}^{\widetilde{L}}$ which do not contain the origin. In particular, $\Phi \widetilde{L}_{\widetilde{A}}^{\widetilde{L}}$ contains the empty face.

Figure 1 shows three $\left(\widetilde{A}(w), \mathcal{F} L_{r}\right)$-umbrellas for $A=(1,4)$ and $w=(-1,1)$. In each case the shaded region is the polyhedron $\Delta \widetilde{\widetilde{L}}_{\widetilde{A}}^{\widetilde{T}}$. For $r=3 / 5$ the point $\frac{\widetilde{a}_{3}}{-r}$ belongs to the line passing through $\widetilde{a}_{1}$ and $\widetilde{a}_{2}$, which means that $s=r+1=8 / 5$ is a slope of the system along $x_{3}=0$. 

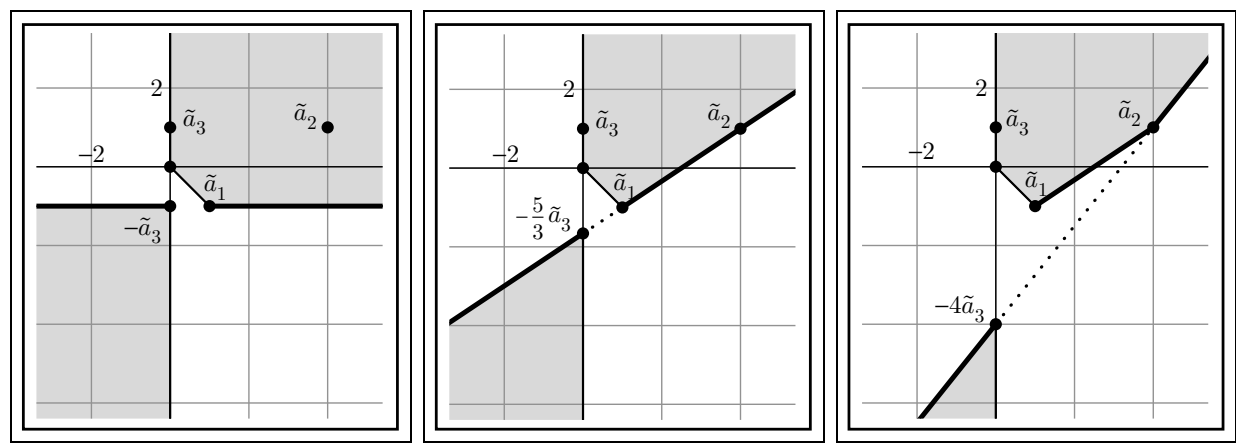

Figure 1. The first umbrella is for $r=1$, the second is for $r=3 / 5$, and the third is for $r=1 / 4$.

Using Definition 1.1, equation (3.2) and [27, Cor. 4.12] we get the following:

Corollary 3.3. The real number $s=r+1>1$ is a slope of $M_{A, w, \alpha}(\beta)$ along $T$ at $p \in T$ if and only if $\Phi_{\widetilde{A}(w)}^{\mathcal{F} L_{r^{\prime}}}$ is not locally constant at $r^{\prime}=r$.

Let us denote by $A_{w}$ the matrix with columns $\widetilde{a}_{i}, 1 \leq i \leq n$, and let $\Delta_{A_{w}}$ be the convex hull of $\left\{\widetilde{a}_{i}: 1 \leq i \leq n\right\}$ and the origin. With this notation, Corollary 3.3 can be rephrased as follows.

Corollary 3.4. The real number $s=r+1>1$ is a slope of $M_{A, w, \alpha}(\beta)$ along $T$ at $p \in T$ if and only if there exists a facet $\tau$ of $\Delta_{A_{w}}$ such that $0 \notin \tau$ and $-\frac{1}{r} \widetilde{a}_{n+1} \in H_{\tau}$, where $H_{\tau}$ is the hyperplane that contains $\tau$.

Remark 3.5. We note that, by the definition of the modified system $M_{A, w, \alpha}(\beta)$, the $D$-modules $M_{A, w, \alpha}(\beta)$ and $M_{A,-w,-\alpha}(\beta)$ agree on $\mathbb{C}^{n} \times \mathbb{C}^{*}$ under the change of the variable $t \rightarrow 1 / t$ in $t \neq 0$. We regard both $D$-modules as extensions of each other. In this paper, when we say the irregularity of $M_{A,-w,-\alpha}(\beta)$ along $T^{\prime}=\{t=\infty\}$, we mean the irregularity of $M_{A, w, \alpha}(\beta)$ along $T=\{t=0\}$. Using this terminology, Corollary 3.4 provides a description of the slopes of the modified system $M_{A, w, \alpha}(\beta)$ along $T^{\prime}$ by using $\Delta_{A_{-w}}$ instead of $\Delta_{A_{w}}$.

Until the end of this section we will denote either $\Phi_{\widetilde{A}}^{v}$ or $\Phi_{\widetilde{A}}^{L}$ for the $(\widetilde{A}, L)-$ umbrella with $L=L_{(u, v)}$ since Definition 3.2 does not depend on $L$ but only on $v \in \mathbb{R}^{n+1}$. Moreover, for any subset $\eta \subseteq\{1, \ldots, n\}$ we denote by $w_{\eta}$ the vector with coordinates equal to the ones of $w$ indexed by $\eta$, i.e., $w_{\eta}=\left(w_{i}\right)_{i \in \eta}$.

In the following two lemmas we assume for simplicity that $w_{i}>0, i=1, \ldots, n$. In fact, this can be assumed without loss of generality since $\widetilde{A}(w)$ is pointed.

Lemma 3.6. For any sufficiently small real number $r>0$, we have

$$
\left\{\eta^{\prime} \in \Phi_{\widetilde{A}(w)}^{\mathcal{F} L_{r}, d}: n+1 \in \eta^{\prime}\right\}=\left\{\sigma \cup\{n+1\}: \sigma \in \Phi_{A_{\eta}}^{w_{\eta}, d-1}, \eta \in \Phi_{A}^{F, d-1}\right\} .
$$

In particular, if all the facets in $\Phi_{A}^{F}$ contain exactly $d$ columns of $A$, then the set of facets of $\Phi_{\widetilde{A}(w)}^{\mathcal{F} L_{r}, d}$ which contain $n+1$ is $\left\{\sigma \cup\{n+1\}: \sigma \in \Phi_{A}^{F, d-1}\right\}$.

Lemma 3.7. For any sufficiently large real number $r>0$ we have that

$$
\Phi_{\widetilde{A}(w)}^{\mathcal{F} L_{r}, d}=\left\{\sigma \cup\{n+1\}: \sigma \in \Phi_{A_{\eta^{\prime}}^{F}}^{F, d-1}, \eta^{\prime} \in \Phi_{A}^{w, d-1}\right\} .
$$


In particular, if all the facets in $\Phi_{A}^{w}$ contain exactly d columns of $A$, then $\Phi_{\widetilde{A}(w)}^{\mathcal{F} L_{r}, d}=$ $\left\{\sigma \cup\{n+1\}: \sigma \in \Phi_{A}^{w, d-1}\right\}$.

Proposition 3.8. The following conditions are equivalent:

(a) $\left\{\sigma \in \Phi_{A_{\eta}}^{w_{\eta}, d-1}: \eta \in \Phi_{A}^{F, d-1}\right\}=\left\{\sigma \in \Phi_{A_{\eta^{\prime}}^{F}}^{F, d-1}, \eta^{\prime} \in \Phi_{A}^{w, d-1}\right\}$.

(b) $\Phi_{\widetilde{A}(w)}^{\mathcal{F} L_{r}}$ is constant for all $r>0$.

(c) $M_{A, w, \alpha}(\beta)$ does not have slopes along $T$.

Proof. Since any umbrella is determined by its facets, Lemma 3.6 and Lemma 3.7 prove that (a) is equivalent to (b). Corollary 3.3 finishes the proof.

Remark 3.9. Note that condition (a) in Proposition 3.8 implies that there is a common refinement (the one given by considering the faces of $\Phi_{\widetilde{A}(w)}^{\mathcal{F} L_{r}}$ not containing $n+1)$ of the polyhedral complex subdivisions induced by the umbrellas $\Phi_{A}^{F}$ and $\Phi_{A}^{w}$. In particular, when $w$ is generic, condition (a) means that $w$ induces a regular triangulation of $A$ that refines the polyhedral complex subdivision induced by the weight vector $(1, \ldots, 1)$. In other words, $w$ is a perturbation of $(1, \ldots, 1)$ for the matrix $A$. For example, this condition is satisfied if either the row span of the matrix $A$ contains the vector $(1, \ldots, 1)$ or $w$ is the sum of a vector in the row span of the matrix $A$ and a vector $(k, \ldots, k)$ with $k \geq 0$.

Remark 3.10. Even when $M_{\widetilde{A}(w)}(\widetilde{\beta})$ is regular holonomic and $M_{A, w, \alpha}(\beta)$ has no slopes along $T$, the latter system is irregular if $M_{A}(\beta)$ is (see Example 3.11).

Example 3.11. Take $A=\left(\begin{array}{ll}1 & 2\end{array}\right)$ and $\beta \in \mathbb{C}$. The system $M_{A}(\beta)$ is irregular along $\left\{x_{2}=0\right\}$ with the unique slope $s=r+1=2$. Put $w=(1,1)$ and

$$
\widetilde{A}(w)=\left(\begin{array}{lll}
1 & 2 & 0 \\
1 & 1 & 1
\end{array}\right) .
$$

The hypergeometric system $M_{\widetilde{A}(w)}(\beta, \alpha-1)$ is regular holonomic for all $\beta, \alpha \in \mathbb{C}$ by a well known result of Hotta [18. However, the modified system $M_{A, w, \alpha}(\beta)$ has a slope $s=r+1=2$ along $T^{\prime}$ because $M_{A,-w,-\alpha}(\beta)$ has the slope $s=2$ along $T$ (see Corollary 3.4 and Remark 3.5).

Remark 3.12. Let $\varphi$ be the map (0.4) defined in the introduction. Since the $\mathcal{D}$ modules $\mathcal{M}_{\widetilde{A}(\mathbf{0})}(\beta,-\alpha)$ and $\mathcal{M}_{A, w, \alpha}(\beta)$ are isomorphic when restricted to $X^{*}=$ $\mathbb{C}^{n} \times \mathbb{C}^{*}$, the slopes of both modules along any coordinate subspace $Z$ not contained in $T$ coincide in $Z \backslash T$. Moreover, the map $\varphi^{*}$ also induces an isomorphism for their spaces of Gevrey solutions along $Z$.

3.3. Holomorphic solutions of a modified hypergeometric system. We study convergent and formal power series solutions of the modified hypergeometric module $\mathcal{M}_{A, w, \alpha}(\beta)$. As was said before, the map $\varphi(0.4)$ induces an isomorphism between the $\mathcal{D}$-modules $\mathcal{M}_{\widetilde{A}(\mathbf{0})}(\beta,-\alpha)$ and $\mathcal{M}_{A, w, \alpha}(\beta)$ when restricted to $X^{*}$, and also an isomorphism between their corresponding spaces of holomorphic solutions. More precisely, for any germ of a holomorphic function $f(x, t)$ at a point $\left(x_{0}, t_{0}\right)$ in $X^{*}$, the function $f(x, t)$ is a solution of $\mathcal{M}_{A, w, \alpha}(\beta)$ if and only if $\varphi^{*}(f)(y, s)=f\left(s^{-w_{1}} y_{1}, \ldots, s^{-w_{n}} y_{n}\right)$ is a germ of a solution of $\mathcal{M}_{\widetilde{A}(\mathbf{0})}(\beta,-\alpha)$ at the 
point $\left(y_{0}, s_{0}\right) \in Y^{*}$ such that $\varphi\left(y_{0}, s_{0}\right)=\left(x_{0}, t_{0}\right)$. We can rewrite this as follows: the morphism

$$
\varphi^{*}: \mathcal{H o m}_{\mathcal{D}_{X^{*}}}\left(\mathcal{M}_{A, w, \alpha}(\beta)_{\mid X^{*}}, \mathcal{O}_{X^{*}}\right) \stackrel{\sim}{\longrightarrow} \mathcal{H o m}_{\mathcal{D}_{Y^{*}}}\left(\mathcal{M}_{\widetilde{A}(\mathbf{0})}(\beta,-\alpha)_{\mid Y^{*}}, \mathcal{O}_{Y^{*}}\right)
$$

is an isomorphism of sheaves of vector spaces. As a consequence the holonomic ranks of both modules coincide,

$$
\operatorname{rank}\left(H_{A, w, \alpha}(\beta)\right)=\operatorname{rank}\left(H_{\widetilde{A}(\mathbf{0})}(\beta,-\alpha)\right),
$$

and this last rank equals the one of $H_{A}(\beta)$ for any $w$, see [30, Theorem 1].

Recall that if $\beta$ is generic, then $\operatorname{rank}\left(H_{A}(\beta)\right)=\operatorname{vol}(A)$, where $\operatorname{vol}(A)$ is the normalized volume of the matrix $A\left[1,14,23,26\right.$, while in general $\operatorname{rank}\left(H_{A}(\beta)\right) \geq$ $\operatorname{vol}(A)[23,26$.

3.4. Gevrey solutions of a modified hypergeometric system. We describe the solutions of $\mathcal{M}_{A, w, \alpha}(\beta)$ in the space $\mathcal{O}_{\widehat{X \mid T}}$ of formal power series with respect to $T=\{t=0\} \subset X=\mathbb{C}^{n+1}$. More generally, we also describe the solutions of $\mathcal{M}_{A, w, \alpha}(\beta)$ in the space $\sum_{\gamma \in \Lambda} t^{\gamma} \mathcal{O}_{\widehat{X \mid T}}$ for any finite set $\Lambda \subseteq \mathbb{C}$ (see Theorem 3.20). We will use notation in [30. Let $\tau$ be the weight vector $(\mathbf{0},-1, \mathbf{0}, 1) \in \mathbb{Z}^{2 n+2}$ inducing the Malgrange-Kashiwara $V$-filtration along $T \equiv(t=0)$ on the ring $D_{n+1}$.

Let $\tilde{I}_{\widetilde{A}(w)} \subseteq \mathbb{C}[\partial, t]:=\mathbb{C}\left[\partial_{1}, \ldots, \partial_{n}, t\right]$ be the toric ideal associated with $\widetilde{A}(w)$, i.e., the binomial ideal generated by the operators in (0.3). The next lemma is technical.

Lemma 3.13. For all $w \in \mathbb{Z}^{n}$ we have $\operatorname{in}_{(\mathbf{0},-1)}\left(\widetilde{I}_{\widetilde{A}(w)}\right)=\mathbb{C}[\partial, t] \operatorname{in}_{w}\left(I_{A}\right)$.

Recall that the indicial polynomial (also called a b-function) of $H_{A, w}(\beta)$ along $T$ is the polynomial $b(s) \in \mathbb{C}[s]$ such that $b\left(\theta_{t}\right)$ is the monic generator of $\operatorname{in}_{\tau}\left(H_{A, w}(\beta)\right) \cap$ $\mathbb{C}\left[\theta_{t}\right]$ where $\theta_{t}=t \partial_{t}$. Moreover, we have by [30, Th. 3] that for $\beta$ and $w$ generic, the indicial polynomial of $H_{A, w}(\beta)$ along $T$ is

$$
b(s)=\prod_{\left(\partial^{\mathbf{k}}, \sigma\right) \in \mathcal{T}(M)}\left(s-w \beta^{\left(\partial^{\mathbf{k}}, \sigma\right)}\right),
$$

where $M=\operatorname{in}_{w}\left(I_{A}\right), \mathcal{T}(M)$ is the set of top-dimensional standard pairs of $M$ (see [26, Sec. 3.2]) and $v=\beta^{\left(\partial^{\mathbf{k}}, \sigma\right)}$ is the vector defined as $v_{i}=k_{i} \in \mathbb{N}$ for $i \notin \sigma$ and $A v=\beta$, which is also an exponent of $H_{A}(\beta)$ with respect to $w$ (see [26, Lemma 4.1.3]).

Definition 3.14. We say that a (generic) vector $\widetilde{w} \in \mathbb{Q}^{n}$ is a (generic) perturbation of $w \in \mathbb{Z}^{n}$, with respect to $A$, if there exists $w^{\prime} \in \mathbb{Q}^{n}$ such that $\operatorname{in}_{\widetilde{w}}\left(I_{A}\right)=$ $\operatorname{in}_{w^{\prime}}\left(\operatorname{in}_{w}\left(I_{A}\right)\right)$.

Remark 3.15. If $\widetilde{w}$ is generic, then $\operatorname{in}_{\widetilde{w}}\left(I_{A}\right)$ is a monomial ideal and it is well known that its degree equals the cardinality of its set of top-dimensional standard pairs $\mathcal{T}\left(\operatorname{in}_{\widetilde{w}}\left(I_{A}\right)\right)$. Moreover, for very generic $\beta \in \mathbb{C}^{d}$ there are exactly $\operatorname{deg}\left(\operatorname{in}_{\widetilde{w}}\left(I_{A}\right)\right)$ many exponents of $H_{A}(\beta)$ with respect to $\widetilde{w}$; see [8, Prop. 4.10].

Lemma 3.16. Let $\beta \in \mathbb{C}^{d}$ be very generic and $w \in \mathbb{Z}^{n}$. There is a generic perturbation $\widetilde{w} \in \mathbb{Q}^{n}$ of $w$ such that for any exponent $v \in \mathbb{C}^{n}$ of $H_{A}(\beta)$ with respect to $\widetilde{w}$, the series $\psi_{v}(x, t)=t^{-\alpha} \phi_{v}\left(t^{w} x\right)$, for $t^{w} x=\left(t^{w_{1}} x_{1}, \ldots, t^{w_{n}} x_{n}\right)$, is a solution of $\mathcal{M}_{A, w, \alpha}(\beta)$ of the form $\psi_{v}(x, t)=\sum_{m \geq 0} f_{m}(x) t^{\gamma+m} \in t^{\gamma} \mathcal{O}_{\widehat{X \mid T},(p, 0)}$, with $\gamma=w v-\alpha$ and $f_{0}(x) \neq 0$ for some $p \in \mathbb{C}^{n}$. 
Proof. Since $\beta$ is very generic, for any generic $\widetilde{w}$ an exponent $v$ of $H_{A}(\beta)$ with respect to $\widetilde{w}$ can be written as $v=\beta^{\left(\partial^{\mathbf{k}}, \sigma\right)}$ where $\left(\partial^{\mathbf{k}}, \sigma\right)$ is a top-dimensional standard pair of $\operatorname{in}_{\widetilde{w}}\left(I_{A}\right)$; see [26, Sec. 3.4]. In particular, $\sigma \in \Phi_{A}^{\widetilde{w}, d-1}, \mathbf{k}=$ $\left(k_{i}\right)_{i \notin \sigma} \in \mathbb{N}^{n-d}, A v=\beta$ and $v_{i}=k_{i} \in \mathbb{N}$ for all $i \notin \sigma$.

The series $\phi_{v}(x)$ is either a holomorphic solution or a Gevrey solution of $\mathcal{M}_{A}(\beta)$ along a coordinate subspace $Z \subseteq \mathbb{C}^{n}$ at any point $p$ in a nonempty relatively open set in $Z$ (see Theorem 2.4 and [10, Th. 3.11] for the details), and since $\beta$ is very generic we have that $N_{v}=\left(-B_{\sigma} \mathbf{k}+\mathbb{N} B_{\sigma}\right) \cap \mathbb{Z}^{n}$.

The expression $f(x, t):=t^{-w v} \phi_{v}\left(t^{w} x\right)=t^{\alpha-w v} \psi_{v}(x, t)$ (resp. $\psi_{v}(x, t)$ ) formally satisfies the equations defining $\mathcal{M}_{A, w, w v}(\beta)$ (resp. $\left.\mathcal{M}_{A, w, \alpha}(\beta)\right)$. We will prove that we can write $f(x, t)=\sum_{m>0} f_{m}(x) t^{m}$ and that it is a Gevrey series along $T \subset X$. Recalling the expression of $\phi_{v}$ in (2.1) it is enough to prove that for all $u \in N_{v} \backslash\{0\}$ we have $w u \in \mathbb{N}$ and that the coefficient of $t^{m}$ in $f$, i.e. $f_{m}(x)=$ $\sum_{u \in N_{v}, w u=m} \frac{[v]_{u_{-}}}{[v+u]_{u_{+}}} x^{v+u}$, is a convergent series in an open neighborhood of some $p \in \mathbb{C}^{n}$, with both the neighborhood and $p$ independent of $m$.

We can take a generic perturbation $\widetilde{w} \in \mathbb{Q}^{n}$ of $w$ of the form $\widetilde{w}=w+\epsilon \widetilde{e}$ with $\widetilde{e}=(1, \ldots, 1)+\epsilon^{\prime} w^{\prime}$ for $\epsilon>0$ and $\epsilon^{\prime}>0$ small enough and $w^{\prime} \in \mathbb{Q}^{n}$ generic.

Take any $u \in N_{v} \backslash\{0\}$ and let us prove that $w u \geq 0$. Since $v$ is an exponent of $H_{A}(\beta)$ with respect to $\widetilde{w}$, we have by [26, (3.30)] that $\widetilde{w} u>0$. Hence, since the last inequality holds for $\epsilon>0$ and $\epsilon^{\prime}>0$ small enough, we have that $w u \geq 0$. Thus we have that $w u \geq 0$ for all $u \in N_{v}$. Notice that when $w u>0$ for all $u \in N_{v} \backslash\{0\}$, then the set $\left\{u \in N_{v}, w u=m\right\}$ is finite and hence $f_{m}(x)$ is clearly convergent. In general, $\left\{u \in N_{v}, w u=m\right\}$ is not finite, but we will see that $f_{m}(x)$ is still convergent at some point $p \in \mathbb{C}^{n}$. Since $N_{v}=\left(-B_{\sigma} \mathbf{k}+\mathbb{N} B_{\sigma}\right) \cap \mathbb{Z}^{n}$ the set $\left\{u \in N_{v}, w u=m\right\}$ is a finite union of shifted copies of the form $N(i)=$ $u(i)+\left(\sum_{j \notin \sigma ; w b_{j}=0} \mathbb{N} b_{j}\right) \cap \mathbb{Z}^{n}$, where $\left\{b_{j} \mid j \notin \sigma\right\}$ is the set of columns of $B_{\sigma}$ and $u(i) \in N_{v}$ satisfies $w u(i)=m$. The series $f_{m}(x)$ is convergent if and only if all the series $g_{i, m}(x)=\sum_{u \in N(i)} \frac{[v]_{u_{-}}}{[v+u]_{u_{+}}} x^{v+u}$ are convergent. Since $\beta$ is very generic, $\operatorname{nsupp}(v)=\emptyset$, and the convergence of each series $g_{i, m}$ is equivalent to the convergence of the series $x^{v+u(i)} \sum_{u \in-u(i)+N(i)} \frac{\left|u_{-}\right| !}{\left|u_{+}\right| !} x^{u}$. Thus, it is enough to see that for any column $u$ of $B_{\sigma}$ such that $w u=0$ we have that $|u|=\left|u_{+}\right|-\left|u_{-}\right| \geq 0$. Notice that $w u=0$ implies $0<\widetilde{w} u=\epsilon\left(|u|+\epsilon^{\prime} w^{\prime} u\right)$ and so $|u|+\epsilon^{\prime} w^{\prime} u>0$. Hence, since this holds for $\epsilon^{\prime}>0$ small enough, we have that $|u| \geq 0$. We have proved that $f$ is a formal solution of $M_{A, w, w v}(\beta)$ along $T$ and it is clear that $f_{0}(x)=x^{v}+\cdots \neq 0$. From the expression of the $g_{i, m}$ and [10, Th. 3.11], any $f_{m}(x)$ is convergent at any point in $\left\{x \in \mathbb{C}^{n}\left|0 \neq \prod_{i \in \sigma} x_{i},\right| x_{j}|<R| x_{\sigma}^{A_{\sigma}^{-1} a_{j}} \mid\right.$ for $j \notin \sigma$ and $\left.\left|w b_{j}\right|=0\right\}$ for some $R>0$.

Let $\widetilde{w} \in \mathbb{Q}^{n}$ be a generic perturbation of $w \in \mathbb{Z}^{n}$ as in the proof of Lemma 3.16.

Lemma 3.17. If $f(x, t)=\sum_{m \geq 0} f_{m}(x) t^{\gamma+m} \in t^{\gamma} \mathcal{O}_{\widehat{X \mid T}, p}$ is a solution of $\mathcal{M}_{A, w, \alpha}(\beta)$ for some $\gamma \in \mathbb{C}, p \in T$, with $f_{0}(x) \neq 0$, then the following hold:

(a) $t^{\alpha} f(x, t)$ is a solution of $\mathcal{M}_{A, w}(\beta)$.

(b) For all $m \geq 0, f_{m}(x)$ is a holomorphic solution of the hypergeometric system $\mathcal{M}_{A_{w}}(\beta, \alpha+\gamma+m)$. 
(c) $b(\alpha+\gamma)=0$, where $b(s)$ is the indicial polynomial of $H_{A, w}(\beta)$ along $T$.

(d) If $\beta$ is very generic, then $\alpha+\gamma=w v$ for some exponent $v$ of $H_{A}(\beta)$ with respect to $\widetilde{w}$.

Proof. The proofs of (a) and (b) are straightforward. Let us prove (c). By (a) and using [26, Theorem 2.5.5] we have that $\operatorname{in}_{(0,1)}\left(t^{\alpha} f(x, t)\right)=f_{0}(x) t^{\alpha+\gamma}$ is a solution of $\operatorname{in}_{\tau}\left(H_{A, w}(\beta)\right)$.

Recall by the definition of $b(s)$ that $\left\langle b\left(\theta_{t}\right)\right\rangle=\operatorname{in}_{\tau}\left(H_{A, w}(\beta)\right) \cap \mathbb{C}\left[\theta_{t}\right]$ where $\theta_{t}=$ $t \partial_{t}$. Thus, $b\left(\theta_{t}\right)$ annihilates $\operatorname{in}_{(0,1)}\left(t^{\alpha} f(x, t)\right)=f_{0}(x) t^{\alpha+\gamma}$. Then we have, using e.g. [26, Lemma 1.3.2], that $0=b\left(\theta_{t}\right)\left(f_{0}(x) t^{\alpha+\gamma}\right)=b(\alpha+\gamma) f_{0}(x) t^{\alpha+\gamma}$, and so $b(\alpha+\gamma)=0$.

Let us prove (d). By (b) we have that $f_{0}(x)$ is a holomorphic solution of $H_{A_{w}}(\beta, \alpha+\gamma)$ and thus it can be written as a Nilsson series at the origin with respect to a vector $\widetilde{e}$ that is a perturbation of $e=(1, \ldots, 1)$ (see [26, 25, [8]). In particular, it makes sense to consider the initial form of $f_{0}(x)$ with respect to $\widetilde{e}$. On the other hand, using Lemma 3.13, we have that $\operatorname{in}_{w}\left(I_{A}\right)+\langle A \theta-\beta\rangle \subseteq \operatorname{in}_{\tau}\left(H_{A, w}(\beta)\right)$ annihilates $f_{0}(x)$. Since $\beta$ is very generic, $\operatorname{in}_{w}\left(I_{A}\right)+\langle A \theta-\beta\rangle=\operatorname{in}_{(-w, w)} H_{A}(\beta)$ [26. Th. 3.1.3]. This implies that $f_{0}(x)$ is a solution of $\operatorname{in}_{(-w, w)} H_{A}(\beta)$ and, hence, $\operatorname{in}_{\widetilde{e}}\left(f_{0}(x)\right)$ is a solution of $\operatorname{in}_{(-\widetilde{w}, \widetilde{w})} H_{A}(\beta)$ for $\widetilde{w}=w+\epsilon \widetilde{e}$. Thus, since $\beta$ is very generic in $\widetilde{e}\left(f_{0}(x)\right)=c x^{v}$ for $c \in \mathbb{C}$ and $v$ is an exponent of $H_{A}(\beta)$ with respect to $\widetilde{w}$. Hence, using (b), we also have that $w v=\alpha+\gamma$.

Remark 3.18. Although we assume in this paper that $\widetilde{A}(w)$ is pointed, it turns out that in this section this fact is only used in the proof of (d) in Lemma 3.17. However, let us notice that if $\widetilde{A}(w)$ is not pointed and $w$ is generic, then $\operatorname{in}_{w}\left(I_{A}\right)=\mathbb{C}[\partial]$, $\operatorname{in}_{\tau}\left(H_{A, w}(\beta)\right)=D$, and so $b(s)=1$. Thus, by (c) in Lemma 3.17 the modified system $\mathcal{M}_{A, w, \alpha}(\beta)$ does not have any solution in $t^{\gamma} \mathcal{O}_{\widehat{X \mid T}, p}$ for all $\gamma \in \mathbb{C}$ and $p \in T$.

Remark 3.19. Since $\widetilde{w}=w+\epsilon \widetilde{e}$ with $\widetilde{e}=(1, \ldots, 1)+\epsilon^{\prime} w^{\prime}$ for sufficiently small $\epsilon>0$ and $\epsilon^{\prime}>0$, we have that $\operatorname{in}_{\widetilde{w}}\left(I_{A}\right)=\operatorname{in}_{w^{\prime}}\left(\operatorname{in}_{e}\left(\operatorname{in}_{w}\left(I_{A}\right)\right)\right)$ for $e=(1, \ldots, 1)$. In particular, $\operatorname{in}_{\widetilde{w}}\left(I_{A}\right)$ and $\operatorname{in}_{w}\left(I_{A}\right)$ have the same degree.

Let us denote by $\operatorname{dim}_{\mathbb{C}}(\mathcal{M}, \mathcal{F})_{p}$ the dimension of the space of $\mathcal{F}$-solutions of a $\mathcal{D}$-module $\mathcal{M}$ at a point $p$.

Theorem 3.20. Let $\beta \in \mathbb{C}^{d}$ be very generic, $\alpha \in \mathbb{C}$ and $v$ an exponent of $H_{A}(\beta)$ with respect to $\widetilde{w}$. Then $\operatorname{dim}_{\mathbb{C}}\left(\mathcal{M}_{A, w, \alpha}(\beta), \mathcal{O}_{\widehat{X \mid T}}\right)_{p}=0$ if $w v-\alpha \notin \mathbb{N}$. Moreover,

$$
\operatorname{dim}_{\mathbb{C}}\left(\mathcal{M}_{A, w, \alpha}(\beta), \sum_{b(\alpha+\gamma)=0} t^{\gamma} \mathcal{O}_{\widehat{X \mid T}}\right)_{p}=\operatorname{deg}\left(\operatorname{in}_{w}\left(I_{A}\right)\right) .
$$

In particular, if $w$ is generic we have $\operatorname{dim}_{\mathbb{C}}\left(\mathcal{M}_{A, w, w v-m}(\beta), \mathcal{O}_{\widehat{X \mid T}}\right)_{p}=1$ for all $p \in T, m \in \mathbb{N}$ and any exponent $v$ of $H_{A}(\beta)$ with respect to $w$.

Proof. The first statement follows from Lemma $3.17(\mathrm{~d})$.

The inequality $\operatorname{dim}_{\mathbb{C}}\left(\mathcal{M}_{A, w, \alpha}(\beta), \sum_{b(\alpha+\gamma)=0} t^{\gamma} \mathcal{O}_{\widehat{X \mid T}}\right)_{p} \geq \operatorname{deg}\left(\operatorname{in}_{w}\left(I_{A}\right)\right)$ follows from Lemma 3.16. Remark 3.15] and Remark 3.19. Moreover, since the operators defining $\mathcal{M}_{A, w, \alpha}(\beta)$ have polynomial coefficients, then any solution $f \in$ $\sum_{b(\alpha+\gamma)=0} t^{\gamma} \mathcal{O}_{\widehat{X \mid T}, p}$ of $\mathcal{M}_{A, w, \alpha}(\beta)$ decomposes as a finite sum of solutions, each of them in a space $t^{\gamma} \mathcal{O}_{\widehat{X \mid T}, p}$. Recall by the proof of Lemma 3.17 that any solution $f \in t^{\gamma} \mathcal{O}_{\widehat{X \mid T}, p}$ of $\mathcal{M}_{A, w, \alpha}(\beta)$ verifies that $\operatorname{in}_{(\widetilde{w}, 0)}\left(\operatorname{in}_{(\mathbf{0}, 1)}(f)\right)=c x^{v} t^{w v-\alpha}$ for 
an exponent $v$ of $H_{A}(\beta)$ with respect to $\widetilde{w}$. This last fact, Remark 3.15, Remark [3.19] and a slightly modified version of [26. Proposition 2.5.7] prove that $\operatorname{dim}_{\mathbb{C}}\left(\mathcal{M}_{A, w, \alpha}(\beta), \sum_{b(\alpha+\gamma)=0} t^{\gamma} \mathcal{O}_{\widehat{X \mid T}}\right)_{p} \leq \operatorname{deg}\left(\operatorname{in}_{w}\left(I_{A}\right)\right)$.

Finally, the last statement follows from the second statement and from the fact that if $\beta$ is very generic, $w \in \mathbb{Z}^{n}$ is generic and $v$ and $v^{\prime}$ are two different exponents of $H_{A}(\beta)$ with respect to $w$, then $w\left(v-v^{\prime}\right) \notin \mathbb{Z}$.

Remark 3.21. Let $\psi_{v}(x, t)$ be the series constructed in Lemma 3.16 and used in Theorem 3.20. If $w$ is in the row span of $A$, then $f(x, t)=t^{\alpha-w v} \psi_{v}$ does not depend on $t$ and thus it is a convergent series. If $w$ is not in the row span of $A$, then $f(x, t)$ is Gevrey along $T$ with index $s=r+1$, where $r=\max \left\{-|u| /(w u): \mathbb{N} u \subseteq N_{v}, w u>\right.$ $0\}$ where $|u|=\sum_{i} u_{i}$. On the other hand, as mentioned in the proof of Lemma 3.16. since $\beta$ is very generic and $v$ is an exponent of $H_{A}(\beta)$ with respect to $\widetilde{w}$, then $v$ is associated with a simplex $\sigma \in \Phi_{A}^{\widetilde{w}, d-1}$ and there is a basis $\left\{b_{i} \mid i \notin \sigma\right\}$ of $\operatorname{ker}(A)$ such that for all $i \notin \sigma,\left(b_{i}\right)_{j}=0$ for all $j \notin \sigma \cup\{i\}$ and $\left(b_{i}\right)_{i}=1$. The set $\left\{b_{i} \mid i \notin \sigma\right\}$ is the set of columns of $B_{\sigma}$ (if we reorder the variables so that $\sigma=\{1, \ldots, d\}$; see Subsection 2.2), and in this case we have that $N_{v}=\left(-B_{\sigma} \mathbf{k}+\mathbb{N} B_{\sigma}\right) \cap \mathbb{Z}^{n}$. Thus, more explicitly, $r=\max \left\{-\left|b_{i}\right| /\left(w b_{i}\right) \mid i \notin \sigma, w b_{i}>0\right\}$, where $\left|b_{i}\right|=1-\left|A_{\sigma}^{-1} a_{i}\right|$ and $w b_{i}=w_{i}-w_{\sigma} A_{\sigma}^{-1} a_{i}>0$. The proof of this formula is technical and follows from standard estimates on $\Gamma$-functions similar to the ones used in [10] to compute the index of Gevrey solutions of hypergeometric systems. In particular, if $w$ is a perturbation of $(1, \ldots, 1)$, then $r$ is close to -1 , and if $A$ is homogeneous, then $r=0$ because $|u|=0$ for any $u \in N_{v}$ and hence in both cases the series is convergent.

3.5. Gevrey solutions modulo convergent series. By Theorem 3.20, if both $\alpha \in \mathbb{C}$ and $\beta \in \mathbb{C}^{d}$ are very generic, then $\mathcal{M}_{A, w, \alpha}(\beta)$ does not have any nonzero solution in $\mathcal{O}_{\widehat{X \mid T}, p}$ for all $p \in T$. This is in contrast with the case of the irregularity of hypergeometric systems along coordinate hyperplanes, where for any slope $s=$ $r+1$ of $\mathcal{M}_{A}(\beta)$ along $Y=\left\{x_{n}=0\right\}$ and for very generic $\beta \in \mathbb{C}^{d}$ one can construct a formal solution $\phi \in \mathcal{O}_{\widehat{X \mid Y}, p}$ of $\mathcal{M}_{A}(\beta)$ along $Y$, such that $\phi$ has Gevrey index equal to the slope (see [10] and Theorem 2.4).

However, by the comparison theorem for the slopes [22] and the perversity of the irregularity complex of a holonomic $\mathcal{D}$-module along a smooth hypersurface 24, one knows that for each slope $s=r+1$ of $\mathcal{M}_{A, w, \alpha}(\beta)$ along $T$ at a generic $p \in T$ there must exist a formal series $\phi \in \mathcal{O}_{\widehat{X \mid T}, p}$ with Gevrey index $s=r+1$ such that $P(\phi)$ is convergent at $p$ for all $P \in H_{A, w, \alpha}(\beta)$.

The purpose of this section is to describe Gevrey solutions modulo convergent series of the modified system $\mathcal{M}_{A, w, \alpha}(\beta)$ along $T$ when $\alpha \in \mathbb{C}$ and $\beta \in \mathbb{C}^{d}$ are very generic. To this end, we use the construction of the Gevrey solutions at infinity of the hypergeometric system $M_{\widetilde{A}(w)}(\beta, \alpha-1)$ as performed in Theorem 2.5 ,

Take $X^{\prime}=\mathbb{C}^{n+1}$ with coordinates $\left(x_{1}, \ldots, x_{n}, z\right)$ and $z=1 / t$ so that $X \cap X^{\prime}=$ $\mathbb{C}^{n} \times \mathbb{C}^{*}$. Denote $T^{\prime}=\{t=\infty\}=\{z=0\} \subseteq X^{\prime}$. We can consider for any $\gamma \in \mathbb{C}$ the $\mathbb{C}$-linear map

$$
\begin{aligned}
\Upsilon_{\gamma}: t^{\gamma} \mathcal{O}_{\widehat{X \mid T}, p} & \longrightarrow t^{-1-\gamma} \mathcal{O}_{\widehat{X^{\prime} \mid T^{\prime}}, p^{\prime}} \\
f=\sum_{m \geq 0} f_{m}(x) t^{\gamma+m} & \longmapsto \Upsilon_{\gamma}(f)=\sum_{m \geq 0} f_{m}(x)[-\gamma-1]_{m} t^{-1-\gamma-m},
\end{aligned}
$$

where $p=\left(p_{1}, \ldots, p_{n}, 0\right) \in T$ and $p^{\prime}=\left(p_{1}, \ldots, p_{n}, \infty\right) \in T^{\prime}$. 
Remark 3.22. Notice that $\Upsilon_{\gamma}$ is an isomorphism if and only if $\gamma \notin \mathbb{Z}_{<0}$. In such a case we also have that $\Upsilon_{\gamma}\left(t^{\gamma} \mathcal{O}_{\widehat{X \mid T}}(s-1)\right)=t^{-1-\gamma} \mathcal{O}_{\widehat{X^{\prime} \mid T^{\prime}}}(s)$ for all $s$. It is also clear that $\Upsilon_{0}\left(\sum_{m \geq 0} f_{m}(x) t^{k+m}\right)=[-1]_{k} \Upsilon_{k}\left(\sum_{m \geq 0} f_{m}(x) t^{k+m}\right)$ for all $k \in \mathbb{N}$.

Theorem 3.23. Assume $\alpha \in \mathbb{C}$ and $\beta \in \mathbb{C}^{d}$ to be very generic. If $s=r+1>1$ is a slope of $\mathcal{M}_{A, w, \alpha}(\beta)$ along $T$, then we can construct $\sum_{\tau} \operatorname{vol}\left(\operatorname{conv}\left(0, \widetilde{a}_{i}: i \in \tau\right)\right)$ Gevrey series that are linearly independent solutions of $\mathcal{M}_{A, w, \alpha}(\beta)$ modulo convergent series and whose Gevrey index is equal to $s=r+1$. Here $\tau$ runs over all the facets of $\Delta_{A_{w}}$ such that $-\frac{1}{r} \widetilde{a}_{n+1} \in H_{\tau}$ and $0 \notin \tau$. Moreover, the classes modulo $\mathcal{O}_{\widehat{X \mid T}}(<s)$ of these Gevrey series form a basis of the solution space of $\mathcal{M}_{A, w, \alpha}(\beta)$ in $\left(\mathcal{O}_{\widehat{X \mid T}}(s) / \mathcal{O}_{\widehat{X \mid T}}(<s)\right)_{p}$ for points $p \in T$ in a relatively open set of $T$.

Proof. The existence of such facets $\tau$ is given by Corollary 3.4. Since $-\frac{1}{r} \widetilde{a}_{n+1} \in H_{\tau}$ and $-r=2-s^{\prime}$ for $s^{\prime}=s+1>2$, we have that $s^{\prime}>2$ is a slope of $\mathcal{M}_{\widetilde{A}(w)}(\beta, \alpha-1)$ along $T^{\prime}=\{t=\infty\}$. Thus, we can construct $\sum_{\tau} \operatorname{vol}\left(\operatorname{conv}\left(0, \widetilde{a}_{i}: i \in \tau\right)\right.$ Gevrey series along $T^{\prime}=\{t=\infty\}$ with index $s^{\prime}$ by Theorem 2.5. Moreover, the classes in $\mathcal{O}_{\widehat{X^{\prime} \mid T^{\prime}}}\left(s^{\prime}\right) / \mathcal{O}_{\widehat{X^{\prime} \mid T^{\prime}}}\left(<s^{\prime}\right)$ are linearly independent solutions of $\mathcal{M}_{\widetilde{A}(w)}(\beta, \alpha-1)$.

More precisely, for any $d$-simplex $\sigma \subseteq \tau$ the series constructed are of the form $\phi_{\widetilde{v}}$ for $\widetilde{v}=(v,-1-k$ ) with $A v=\beta, w v-1-k=\alpha-1$ (i.e. $w v-\alpha=k \in \mathbb{N}$ ) and $v_{i} \in \mathbb{N}$ for all $i \in\{1, \ldots, n\} \backslash \sigma$.

Using Remark 3.22 we can take $\psi_{v}(x, t)$ as the unique Gevrey series along $T$ with index $s=r+1$ verifying $\Upsilon_{0}\left(\psi_{v}(x, t)\right)=\phi_{\widetilde{v}}$ for $\widetilde{v}=(v,-1-k)$.

We conclude by Remark 3.22 that the $\sum_{\tau} \operatorname{vol}\left(\operatorname{conv}\left(0, \widetilde{a}_{i}: i \in \tau\right)\right.$ series $\psi_{v}(x, t)$ constructed are Gevrey series with index $s=s^{\prime}-1=r+1$ whose classes modulo $\mathcal{O}_{\widehat{X \mid T}}(<s)$ are linearly independent. Moreover, it can be checked that they are solutions of the modified system modulo $\mathcal{O}_{X \mid T}$ by using the fact that their images by the morphism $\Upsilon_{0}$ are solutions of $\mathcal{M}_{\widetilde{A}(w)}(\beta, \alpha-1)$.

Last statement follows from (3.1), [22] and [27].

Example 3.24. Take $A=\left(\begin{array}{lll}1 & 3 & 5\end{array}\right), w=(0,1,1)$ and $\beta, \alpha \in \mathbb{C}$. We have that $\widetilde{I}_{\widetilde{A}(w)}=\left\langle\partial_{2}-t \partial_{1}^{3}, \partial_{3}-t \partial_{1}^{5}\right\rangle$ and $H_{A, w, \alpha}(\beta)=D \widetilde{I}_{\widetilde{A}(w)}+D\left\langle x_{1} \partial_{1}+3 x_{2} \partial_{2}+5 x_{3} \partial_{3}-\right.$ $\left.\beta, x_{2} \partial_{2}+x_{3} \partial_{3}-t \partial_{t}-\alpha\right\rangle$. Note here that $\widetilde{I}_{\widetilde{A}(w)}$ is the binomial ideal generated by the operators in (0.3). The unique slope of $M_{A, w, \alpha}(\beta)$ along $T$ is $s=r+1=5$ since $-\frac{1}{4} \widetilde{a}_{4}$ belongs to the line passing through $\widetilde{a}_{1}, \widetilde{a}_{3}$ and $\sigma=\{1,3\}$ is a facet of $\Phi_{\widetilde{A}(w)}^{\mathcal{F} L_{r}}$ if and only if $r \geq 4$.

The volume of $\sigma$ is one, and following the proofs of Theorem 3.23 and Theorem 2.5 we can take $v=(\beta-5 \alpha, 0, \alpha)$ which satisfies the conditions $w v-\alpha=k=0 \in \mathbb{N}$, $v_{2}=0 \in \mathbb{N}$ and $A v=\beta$. We get that the series

$$
\psi_{v}(x, t)=\sum_{m_{2}, m_{2}+m_{3} \geq 0} \frac{[\beta-5 \alpha]_{3 m_{2}+5 m_{3}}}{\left[\alpha+m_{3}\right]_{m_{3}} m_{2} !} x_{1}^{\beta-5 \alpha-3 m_{2}-5 m_{3}} x_{2}^{m_{2}} x_{3}^{\alpha+m_{3}} t^{m_{2}+m_{3}}
$$

is a Gevrey solution (modulo convergent series) of $M_{A, w, \alpha}(\beta)$ along $T$ with index $s=r+1=5$. 


\section{Borel transformation AND ASYMPtotic EXPANSION}

We assume that the $\mathbb{Q}$-row span of the matrix $A$ does not contain the vector $(1, \ldots, 1)$, but the one of $A_{w}$ does, where $A_{w}$ is the matrix with columns $\widetilde{a}_{i}, 1 \leq$ $i \leq n$ (see Subsection 3.2).

Solutions in this case can be analyzed by utilizing the Borel transformation and the Laplace transformation. We review here some basics of the Borel summation method which we require in the following (see [3] for the details). Let us consider

$$
f(t)=\sum_{\ell=0}^{\infty} f_{\ell} t^{\ell+\gamma} \in t^{\gamma} \mathbb{C}[[t]]
$$

where $f_{0} \neq 0$ and $\gamma \in \mathbb{C}$. Solutions constructed in Theorem 3.20 are of this form. If its coefficients satisfy

$$
\left|f_{\ell}\right| \leq C K^{\ell} \Gamma(1+(\ell+\gamma) / \kappa) \quad(\ell=0,1,2, \cdots)
$$

with some positive constants $C, K, \kappa$, and $\Re \gamma>-\kappa$ (in (4.9) this last condition will be relaxed), then the formal Borel transform (with index $\kappa$ ) defined by

$$
\hat{\mathcal{B}}_{\kappa}[f](\tau):=\sum_{\ell=0}^{\infty} \frac{f_{\ell}}{\Gamma(1+(\ell+\gamma) / \kappa)} \tau^{\ell+\gamma}
$$

is the product of $\tau^{\gamma}$ and a convergent power series at $\tau=0$. In addition to (4.2), if

(i) the function $\hat{\mathcal{B}}_{\kappa}[f]$ can be analytically continued to a sector

$$
S(\theta, \delta):=\left\{r e^{i \theta^{\prime}} ;\left|\theta^{\prime}-\theta\right|<\delta / 2, r>0\right\}
$$

of infinite radius in a direction $\theta \in \mathbb{R}$ with an opening angle $\delta>0$, and

(ii) the analytic continuation of $\hat{\mathcal{B}}_{\kappa}[f]$ satisfies the growth estimate

$$
\left|\hat{\mathcal{B}}_{\kappa}[f](\tau)\right| \leq c_{1} \exp \left[c_{2}|\tau|^{\kappa}\right]
$$

in $S(\theta, \delta)$ with some positive constants $c_{1}, c_{2}>0$,

then we say $f$ is $\kappa$-summable in the direction $\theta$, and define the $\kappa$-sum (or the Borel sum with index $\kappa$ ) of $f$ by the Laplace transformation

$$
\mathcal{S}[f](t)=\mathcal{L}_{\kappa}^{\theta} \hat{\mathcal{B}}_{\kappa}[f](t):=\int_{0}^{e^{i \theta} \cdot \infty} e^{-(\tau / t)^{\kappa}} \hat{\mathcal{B}}_{\kappa}[f](\tau) \frac{\kappa \tau^{\kappa-1}}{t^{\kappa}} d \tau .
$$

Remark 4.1. Because of the growth condition (ii) of $\hat{\mathcal{B}}_{\kappa}[f]$, the Laplace integral (4.4) converges if $t$ satisfies

$$
\Re\left[\left(\frac{\tau}{t}\right)^{\kappa}\right]-c_{2}|\tau|^{\kappa}>0
$$

Since

$$
\Re\left[\left(\frac{\tau}{t}\right)^{\kappa}\right]-c_{2}|\tau|^{\kappa}=\left|\frac{\tau}{t}\right|^{\kappa}\left\{\cos \kappa(\theta-\arg t)-c_{2}|t|^{\kappa}\right\}
$$

(note that $\arg \tau=\theta$ ), the Laplace integral (4.4) converges in

$$
\left\{t ; \cos [\kappa(\arg t-\theta)]>c_{2}|t|^{\kappa}\right\} .
$$

The region (4.6) has infinitely many connected components. Here and in what follows we specify one of them by imposing $|\arg t-\theta|<\pi /(2 \kappa)$. Since we can vary 
$\arg \tau$ in (4.4) slightly, we conclude that $\mathcal{S}[f]$ defines a holomorphic function in

$$
\bigcup_{\left|\theta^{\prime}-\theta\right|<\delta / 2}\left\{t ; \cos \left[\kappa\left(\arg t-\theta^{\prime}\right)\right]>c_{2}|t|^{\kappa},\left|\arg t-\theta^{\prime}\right|<\pi /(2 \kappa)\right\} .
$$

Therefore we can find $\rho>0$ and $\varpi>\pi / \kappa$ such that the $\mathcal{S}[f]$ is holomorphic in $S(\theta, \varpi, \rho):=S(\theta, \varpi) \cap\{t ; 0<|t|<\rho\}$ (cf. [3, the first paragraph of $\S 2.1]$ ).

Theorem 4.2. If $f$ is $\kappa$-summable in a direction $\theta$, then $f$ is a Gevrey asymptotic expansion of its Borel sum $\mathcal{S}[f](t)$ : For any closed subsector $\bar{S}$ of $S(\theta, \varpi, \rho)$, there exists $C^{\prime}, K^{\prime}>0$ for which the inequality

$$
\left|t^{-\gamma} \mathcal{S}[f](t)-\sum_{\ell=0}^{N-1} f_{\ell} t^{\ell}\right| \leq C^{\prime}\left(K^{\prime}\right)^{N}|t|^{N} \Gamma(1+N / \kappa)
$$

holds in $\bar{S}$ for $N \in \mathbb{N}$.

Proof. Here we give a sketch of the proof. See [3, Th. 1] for the details.

It follows from the relation

$$
t^{\ell+\gamma}=\int_{0}^{e^{i \theta} \cdot \infty} e^{-(\tau / t)^{\kappa}} \frac{\tau^{\ell+\gamma}}{\Gamma(1+(\ell+\gamma) / \kappa)} \frac{\kappa \tau^{\kappa-1}}{t^{\kappa}} d \tau \quad\left(=\mathcal{L}_{\kappa}^{\theta} \hat{\mathcal{B}}_{\kappa}\left[t^{\ell+\gamma}\right]\right)
$$

that the remainder of the expansion becomes

$$
\begin{aligned}
\mathcal{S}[f](t) & -\sum_{\ell=0}^{N-1} f_{\ell} t^{\ell+\gamma} \\
= & \int_{0}^{e^{i \theta} \cdot \infty} e^{-(\tau / t)^{\kappa}}\left\{\hat{\mathcal{B}}_{\kappa}[f](\tau)-\sum_{\ell=0}^{N-1} \frac{f_{\ell}}{\Gamma(1+(\ell+\gamma) / \kappa)} \tau^{\ell+\gamma}\right\} \frac{\kappa \tau^{\kappa-1}}{t^{\kappa}} d \tau .
\end{aligned}
$$

Since

$$
\frac{1}{\tau^{N+\gamma}}\left\{\hat{\mathcal{B}}_{\kappa}[f](\tau)-\sum_{\ell=0}^{N-1} \frac{f_{\ell}}{\Gamma(1+(\ell+\gamma) / \kappa)} \tau^{\ell+\gamma}\right\}
$$

is holomorphic in some neighborhood of $\bar{S}$ (which includes the origin) and satisfies the growth condition (4.3) in $\bar{S}$ with appropriate constants $c_{1}$ and $c_{2}$, the righthand side of (4.8) can be estimated by the right-hand side of (4.7) multiplied by $|t|^{\gamma}$.

Inequality (4.7) is also known to be equivalent to conditions (i) and (ii) stated above ([3, Theorem 1 (p. 23)]).

The Borel summation method may also be applied to the case when

$$
\gamma \notin \kappa \mathbb{Z} \text { and } \ell+\gamma \notin-\kappa \mathbb{N}_{>0} \text { for } \ell \in \mathbb{N} \text {. }
$$

We can define the Borel transform $\hat{\mathcal{B}}_{\kappa}[f]$ in the same manner as before. In this last case, however, the Laplace integral (4.4) may not converge at $\tau=0$. Therefore we modify the definition of the Borel sum to

$$
\mathcal{S}[f](t)=\mathcal{L}_{\kappa}^{\theta} \hat{\mathcal{B}}_{\kappa}[f](t):=\frac{1}{1-e^{-2 \pi i \gamma / \kappa}} \int_{\Gamma_{\kappa \theta}} e^{-\zeta / t^{\kappa}} \hat{\mathcal{B}}_{\kappa}[f]\left(\zeta^{1 / \kappa}\right) \frac{d \zeta}{t^{\kappa}}
$$

with a path of integration $\Gamma_{\kappa \theta}$ which runs from $\infty$ along $\arg \zeta=\kappa \theta-2 \pi$ to some point near the origin, takes a $2 \pi$ radian turn along a circle with the center at the origin, and goes back to infinity in the direction $\arg \zeta=\kappa \theta$. When condition (4.9) 
is satisfied and $\Re \gamma>-\kappa$, then (4.10) coincides with (4.4). The Borel sum (4.10) also satisfies the same properties as previously defined (4.4).

We apply the Borel summation method to the Gevrey solution constructed in Theorem 3.20. Our main result of this section is

Theorem 4.3. We assume that the $\mathbb{Q}$-row span of the matrix $A$ does not contain the vector $(1,1, \ldots, 1)$ but that the one of $A_{w}$ does. We also assume $\beta$ to be very generic. Let

$$
\psi(x, t)=\sum_{\ell=0}^{\infty} C_{\ell}(x) t^{\ell+\gamma}
$$

be one of the formal solutions of the modified hypergeometric system $H_{A, w}(\beta)$ constructed in Theorem 3.20 and $r+1$ be the Gevrey index of $\psi(x, t)$ along $T$. We also assume $r \gamma \notin \mathbb{Z}$. Then the formal solution $\psi(x, t)$ is $1 / r$-summable (as a formal power series in $t$ ) in all but finitely many directions for each $x \in U$, where $U$ is a non-empty open set in the $x$-space $\mathbb{C}^{n}$. Furthermore its Borel sum determines a solution of the modified hypergeometric system $H_{A, w}(\beta)$.

Remark 4.4. Under the assumption of Theorem 4.3 ,

$$
r=-\frac{\left|b_{i}\right|}{w \cdot b_{i}}
$$

holds for any $i \notin \sigma$, where $\left\{b_{i}: i \notin \sigma\right\}$ is the basis of Ker $A$ given in Remark 3.21, Therefore if $u \in \operatorname{Ker} A \cap \mathbb{Z}^{n}$, then $r w \cdot u$ is an integer.

Remark 4.5. The condition that $A_{w}$ contains $(1,1, \ldots, 1)$ is assumed so that the Borel transformed series satisfies a regular holonomic system [18. Hence, the growth condition (ii) of the Borel summability is satisfied because solutions of regular holonomic systems satisfy a polynomial growth condition. Without this assumption, things become more complicated. See also Section 5 .

Proof of Theorem 4.3. First of all, the open set $U$ in the theorem can be chosen as follows. There exist constants $c_{i j}, c_{i}, p_{i}, m$ such that the series $\varphi_{B}(x, \zeta)$, which will be defined in the proof below, converges when $\left(x_{1}, \ldots, x_{n}, \zeta\right)$ belongs to the nonempty open set $W=W^{\prime} \cap\left(\bigcap_{j=1}^{n}\left(x_{j} \neq 0\right)\right)$, where $W^{\prime}$ is defined by the inequalities $\sum_{j} c_{i j} \log \left|x_{j}\right|+c_{i} \log |\zeta|<p_{i}$, for $i=1, \ldots, m$. Such constants exist because $\varphi_{B}(x, \zeta)$ is a hypergeometric series which satisfies a regular holonomic $A$ hypergeometric system ([14], [26, Section 2.5]); see also the forthcoming Lemma 4.7. Since only nonnegative powers of $\zeta$ modulo an exponent appear in $\varphi_{B}$, we may assume that $c_{i}>0$ for $i=1, \ldots, m$. We may choose a nonempty domain $U \subset \mathbb{C}^{n}$ with compact closure such that $U \times\{\zeta \in \mathbb{C}|0<| \zeta \mid<\epsilon\} \subset W$ for some $\epsilon>0$.

To study the analytic properties of $\hat{\mathcal{B}}_{1 / r}[\psi]$, it is convenient to use

$$
\varphi(x, z):=\left.\psi(x, t)\right|_{t=z^{r}}=\sum_{\ell=0}^{\infty} C_{\ell}(x) z^{r(\ell+\gamma)} .
$$

Since $t^{-\gamma} \psi(x, t)$ is a formal power series in $t^{w \cdot b_{i}}, z^{-r \gamma} \varphi(x, z)$ does not contain any fractional powers in $z$ (cf. Remark 4.4). Then we have

$$
\hat{\mathcal{B}}_{1}[\varphi](x, \zeta)=\sum_{\ell=0}^{\infty} \frac{C_{\ell}(x)}{\Gamma(1+r(\ell+\gamma))} \zeta^{r(\ell+\gamma)}=\hat{\mathcal{B}}_{1 / r}[\psi]\left(x, \zeta^{r}\right) .
$$


In what follows we simply write $\varphi_{B}(x, \zeta)$ (resp., $\left.\psi_{B}(x, \tau)\right)$ instead of $\hat{\mathcal{B}}_{1}[\varphi](x, \zeta)$ (resp., $\left.\hat{\mathcal{B}}_{1 / r}[\psi](x, \tau)\right)$.

The following well-known lemma can be proved by exchanging differentiation and summation.

Lemma 4.6. Assume condition (4.9) holds for $\kappa=1 / r$. For the power series $\varphi$ given in (4.13), we have

$$
\theta_{\zeta} \varphi_{B}=\hat{\mathcal{B}}_{1}\left[\theta_{z} \varphi\right] \quad \text { and } \quad \frac{\partial \varphi_{B}}{\partial \zeta}=\hat{\mathcal{B}}_{1}\left[z^{-1} \varphi\right]
$$

Lemma 4.7. The formal power series $\varphi_{B}(x, \zeta)$ given in (4.14) formally satisfies the hypergeometric system $H_{A_{B}}\left(\beta_{B}\right)$, where

$$
A_{B}=\left(\begin{array}{cc}
A & 0 \\
w & -1 / r
\end{array}\right), \quad \beta_{B}=\left(\begin{array}{c}
\beta \\
0
\end{array}\right) .
$$

When the matrix $A_{B}$ contains a rational entry, we regard the $\mathbb{Z}$-module generated by the column vectors as the lattice to define the $A$-hypergeometric system.

Proof. It follows from Lemma 4.6 and relations $\theta_{j} \varphi=\left.\theta_{j} \psi\right|_{t=z^{r}}$ and $\theta_{z} \varphi(x, z)=$ $\left.r\left(\theta_{t} \psi\right)\right|_{t=z^{r}}$ that

$$
\begin{aligned}
\left(\sum_{j=1}^{n} a_{i j} \theta_{j}-\beta\right) \varphi_{B} & =\hat{\mathcal{B}}_{1}\left[\left(\sum_{j=1}^{n} a_{i j} \theta_{j}-\beta\right) \varphi\right] \\
& =\hat{\mathcal{B}}_{1}\left[\left.\left(\sum_{j=1}^{n} a_{i j} \theta_{j}-\beta\right) \psi\right|_{t=z^{r}}\right]=0
\end{aligned}
$$

and

$$
\begin{aligned}
\left(\sum_{i=1}^{n} w_{i} \theta_{i}-\frac{1}{r} \theta_{\zeta}\right) \varphi_{B} & =\hat{\mathcal{B}}_{1}\left[\left(\sum_{i=1}^{n} w_{i} \theta_{i}-\frac{1}{r} \theta_{z}\right) \varphi\right] \\
& =\hat{\mathcal{B}}_{1}\left[\left.\left(\sum_{i=1}^{n} w_{i} \theta_{i}-\theta_{t}\right) \psi\right|_{t=z^{r}}\right]=0 .
\end{aligned}
$$

Now we take vectors $u^{\prime}:=\left(u, u_{n+1}\right)=\left(u_{1}, \ldots, u_{n}, u_{n+1}\right)^{T}, v^{\prime}:=\left(v, v_{n+1}\right)=$ $\left(v_{1}, \ldots, v_{n}, v_{n+1}\right)^{T} \in \mathbb{N}^{n+1}$ satisfying $A_{B} u^{\prime}=A_{B} v^{\prime}$. By its definition, we obtain $(1 / r)\left(u_{n+1}-v_{n+1}\right)=w \cdot(u-v) \in \mathbb{Z}$. Without loss of generality we may assume one of $u_{n+1}$ and $v_{n+1}$ is zero. Under this assumption, $u_{n+1} / r$ and $v_{n+1} / r$ are nonnegative integers. Furthermore

$$
w \cdot u-u_{n+1} / r=w \cdot v-v_{n+1} / r \Longleftrightarrow w \cdot u+v_{n+1} / r=w \cdot v+u_{n+1} / r
$$

holds. Therefore $\widetilde{u}:=\left(u, v_{n+1} / r\right)^{T}, \widetilde{v}:=\left(v, u_{n+1} / r\right)^{T} \in \mathbb{N}^{n+1}$ and $\widetilde{A} \widetilde{u}=\widetilde{A} \widetilde{v}$. Hence

$$
\begin{aligned}
& \left(\partial^{u} \partial_{\zeta}^{u_{n+1}}-\partial^{v} \partial_{\zeta}^{v_{n+1}}\right) \varphi_{B}=\hat{\mathcal{B}}_{1}\left[\left(\partial^{u} z^{-u_{n+1}}-\partial^{v} z^{-v_{n+1}}\right) \varphi\right] \\
& \quad=\hat{\mathcal{B}}_{1}\left[z^{-\left(u_{n+1}+v_{n+1}\right)}\left(\partial^{u} z^{v_{n+1}}-\partial^{v} z^{u_{n+1}}\right) \varphi\right] \\
& \quad=\hat{\mathcal{B}}_{1}\left[\left.z^{-\left(u_{n+1}+v_{n+1}\right)}\left(\partial^{u} t^{v_{n+1} / r}-\partial^{v} t^{u_{n+1} / r}\right) \psi\right|_{t=z^{r}}\right]=0 .
\end{aligned}
$$

Here we have used the second relation of Lemma 4.6. This completes the proof. 
Under the assumption of Theorem 4.3, $H_{A_{B}}\left(\beta_{B}\right)$ is regular holonomic [18]. Therefore, up to a nonzero constant, $\varphi_{B}(x, \zeta)$ is nothing but a GKZ series solution of $H_{A_{B}}\left(\beta_{B}\right)$, and $\varphi_{B}(x, \zeta)$ converges near $\zeta=0$ if $x \in U$. It also follows that the restriction of $\varphi_{B}(x, \zeta)$ to $\left\{x=x^{0}\right\}$, which is a function of $\zeta$, satisfies some linear ordinary differential equation $E\left(x^{0}\right)$ of Fuchsian type. Let $\operatorname{Sing}\left(x^{0}\right)$ be the set of singular points of $E\left(x^{0}\right)$ except the origin and infinity and define $\Theta\left(x^{0}\right)=\left\{\arg u ; u \in \operatorname{Sing}\left(x^{0}\right)\right\}$. For any $\theta \in \mathbb{R}$ with $\theta \notin \Theta\left(x^{0}\right)$ we have:

(i) $\varphi_{B}\left(x^{0}, \zeta\right)$ can be analytically continued to a sector $S(\theta, \delta)$ with some small $\delta>0$ since there are no singular points on $\{\zeta ; \arg \zeta=\theta\}$.

(ii) The Borel transform $\varphi_{B}\left(x^{0}, \zeta\right)$ has polynomial growth with respect to $\zeta$ in $S(\theta, \delta)$ since a singular point of $E\left(x^{0}\right)$ is a regular singular point.

Hence we conclude that $\varphi\left(x^{0}, z\right)$ is Borel summable (i.e., 1-summable) in the direction $\theta$. Since $\Theta\left(x^{0}\right)$ is a finite set for each fixed $x^{0} \in U, \varphi(x, z)$ is Borel summable in all directions in an open interval in $\mathbb{R}$ for all $x$ in a small enough open set that we still denote by $U$. Because of the relation (4.14), $\psi$ is $1 / r$-summable if and only if $\varphi$ is 1 -summable. Therefore $\psi(x, t)$ is $1 / r$-summable in all the directions in an open interval for all points $x \in U$.

Finally we show that the Borel sum of $\psi(x, t)$ is a solution of the modified hypergeometric system $H_{A, w}(\beta)$ to finish the proof of Theorem 4.3. Because of the relation

$$
\left.\mathcal{S}[\psi]\right|_{t=z^{r}}=\left.\mathcal{L}_{1 / r}^{\theta} \hat{\mathcal{B}}_{1 / r}[\psi]\right|_{t=z^{r}}=\mathcal{L}_{1}^{\theta / r} \hat{\mathcal{B}}_{1}[\varphi]=\mathcal{S}[\varphi],
$$

Lemma 4.6 and Lemma 4.7, it is enough to prove (we omit writing $\theta / r$ )

Lemma 4.8 .

$$
z \frac{\partial}{\partial z} \mathcal{L}_{1}\left[\varphi_{B}\right]=\mathcal{L}_{1}\left[\zeta \frac{\partial \varphi_{B}}{\partial \zeta}\right], \quad z^{-1} \mathcal{L}_{1}\left[\varphi_{B}\right]=\mathcal{L}_{1}\left[\frac{\partial \varphi_{B}}{\partial \zeta}\right]
$$

Proof. To begin with, we give a proof in the case when $\Re(r \gamma)>0$ or $\gamma=0$. By differentiating under the integral sign we obtain

$$
\begin{aligned}
z \frac{\partial}{\partial z} \mathcal{L}_{1}\left[\varphi_{B}\right] & =\int_{0}^{\infty} \frac{\zeta}{z} e^{-\zeta / z} \varphi_{B}(x, \zeta) \frac{d \zeta}{z}-\int_{0}^{\infty} e^{-\zeta / z} \varphi_{B}(x, \zeta) \frac{d \zeta}{z} \\
& =-\int_{0}^{\infty} \frac{\partial}{\partial \zeta}\left(\zeta e^{-\zeta / z}\right) \cdot \varphi_{B}(x, \zeta) \frac{d \zeta}{z}
\end{aligned}
$$

By integral by parts, this equals

$$
-\left[\zeta e^{-\zeta / z} \cdot \frac{\varphi_{B}(x, \zeta)}{z}\right]_{\zeta=0}^{\infty}+\int_{0}^{\infty} e^{-\zeta / z} \cdot \zeta \frac{\partial \varphi_{B}}{\partial \zeta}(x, \zeta) \frac{d \zeta}{z} .
$$

Since the boundary terms vanish (the boundary term coming from infinity vanishes because of the growth estimate of $\varphi_{B}$ ), the first relation follows. In a similar manner, we obtain

$$
\begin{aligned}
\mathcal{L}_{1}\left[\frac{\partial \varphi_{B}}{\partial \zeta}\right] & =\int_{0}^{\infty} e^{-\zeta / z} \frac{\partial \varphi_{B}}{\partial \zeta}(x, \zeta) \frac{d \zeta}{z} \\
& =\left[e^{-\zeta / z} \frac{\varphi_{B}(x, \zeta)}{z}\right]_{\zeta=0}^{\infty}+\frac{1}{z} \int_{0}^{\infty} e^{-\zeta / z} \varphi_{B}(x, \zeta) \frac{d \zeta}{z} .
\end{aligned}
$$

The growth estimate of $\varphi_{B}$ at infinity and the behavior of $\varphi_{B}$ near the origin guarantee that the boundary terms vanish. This proves the second relation. 
When $\Re(r \gamma) \leq 0$ and $\gamma$ satisfies (4.9), we use (4.10) as the definition of the Borel sum, and the same argument works. In this case all of the boundary terms come from infinity and they vanish.

This finishes the proof of Theorem 4.3.

In the preceding proof, we have shown that $\psi_{B}(x, \tau)$ is of polynomial growth in $\tau$ for $x \in U$. Therefore, for an arbitrarily small positive $c_{2}$ we can find $c_{1}>0$ for which (4.3) holds with $f=\psi, \kappa=1 / r$ for all $x \in U$ after eventually replacing $U$ by a smaller open set. Therefore, condition (4.6) guarantees the following corollary.

Corollary 4.9. The Laplace integral (4.4) of the Borel transform $\psi_{B}(x, \tau)$ converges in $S(\theta, r \pi)$ if $\theta \notin \Theta(x)$. In particular, we can set $t=1$ in the expression $\mathcal{S}[\psi](x, t)$ of the Borel sum of $\psi(x, t)$ if $\Theta(x)$ does not contain 0 . The series $\mathcal{S}[\psi](x, 1)$ gives a holomorphic solution of $H_{A}(\beta)$ and the formal series $\psi(x, 1)$ also expresses the asymptotic expansion of the solution $\mathcal{S}[\psi](x, 1))$ along a curve $x(t)=\left(t^{w_{1}} x_{1}^{0}, \ldots, t^{w_{n}} x_{n}^{0}\right)$ as $t \rightarrow 0$ for $x^{0} \in U$. That is,

$$
\left|\mathcal{S}[\psi](x(t), 1)-\psi_{N}(x(t), 1)\right| \leq C K^{N}|t|^{N} \Gamma(1+r N)
$$

for all $N \geq 0$ for some constants $C>0, K>0$, where $\psi_{N}(x, t):=\sum_{\ell<N} C_{\ell}(x) t^{\ell}$.

Remark 4.10. Since the singular points $\operatorname{Sing}(x)$ of $E(x)$ depend on $x$, one of them may meet the path of integration of the Borel sum if $x$ moves. In that case we obtain the analytic continuation of the Borel sum by deforming the path of integration. This is closely related to the Stokes phenomenon.

Example 4.11. Put $A=(1,2)$ and $w=(0,1)$. A formal solution of the modified system $H_{A, w}(\beta)$ is

$$
\psi(x, t)=x_{1}^{\beta} \sum_{m=0}^{\infty} \frac{[\beta]_{2 m}}{m !}\left(\frac{x_{2}}{x_{1}^{2}}\right)^{m} t^{m} .
$$

If $\beta \notin \mathbb{N}$, the Gevrey index $s=r+1$ of $\psi(x, t)$ along $t=0$ is $s=2$. We set $z=t$ and $\varphi(x, z)=\psi(x, z)$. The Borel transform $\varphi_{B}$ is

$$
x_{1}^{\beta} \sum_{m=0}^{\infty} \frac{[\beta]_{2 m}}{m !}\left(\frac{x_{2}}{x_{1}^{2}}\right)^{m} \frac{\zeta^{m}}{m !}=x_{1}^{\beta} \cdot{ }_{2} F_{1}\left(-\beta / 2,(-\beta+1) / 2,1 ; 4 x_{2} \zeta / x_{1}^{2}\right) .
$$

The domain $U$ may be defined by $\left\{\left(x_{1}, x_{2}\right)|-2 \log | x_{1}|+\log | x_{2}|<-1,| x_{1} \mid<1\right\}$. The series $\varphi_{B}$ satisfies the $A$-hypergeometric system with $A_{B}=\left(\begin{array}{ccc}1 & 2 & 0 \\ 0 & 1 & -1\end{array}\right)$ and $\beta_{B}=(\beta, 0)^{T}$. The equation $E(x)=E\left(x_{1}, x_{2}\right)$ in the proof is

$$
\left[\left(4 x_{2} \zeta^{2}-x_{1}^{2} \zeta\right)\left(\frac{\partial}{\partial \zeta}\right)^{2}+\left((-4 \beta+6) x_{2} \zeta-x_{1}^{2}\right) \frac{\partial}{\partial \zeta}+\left(\beta^{2}-\beta\right) x_{2}\right] \varphi_{B}(x, \zeta)=0,
$$

and Sing $(x)=\left\{x_{1}^{2} /\left(4 x_{2}\right)\right\}, \Theta(x)=\left\{2 \arg x_{1}-\arg x_{2}\right\}$. The Borel sum of $\psi(x, t)$ is

$$
\frac{x_{1}}{t} \int_{0}^{e^{i \theta} \cdot \infty} e^{-\tau / t}{ }_{2} F_{1}\left(-\beta / 2,(-\beta+1) / 2,1 ; 4 x_{2} \tau / x_{1}^{2}\right) d \tau
$$

and, for each $x \in U, \varphi$ (and hence $\psi$ ) is Borel summable in all directions except the angle $\theta=2 \arg x_{1}-\arg x_{2}$. 
The series $\psi(x, 1)$ can be regarded as an asymptotic expansion of a solution of the original $A$-hypergeometric system for $A=(1,2)$ and $\beta$ from (4.7) and Corollary 4.9. In other words, we have

$$
x_{1}^{\beta} \int_{0}^{e^{i \theta} \infty} e^{-\tau}{ }_{2} F_{1}\left(\frac{-\beta}{2}, \frac{-\beta+1}{2}, 1 ; \frac{4 x_{2} \tau}{x_{1}^{2}}\right) d \tau \sim \psi(x, 1),
$$

which is a well-known asymptotic expansion.

Example 4.12. Put $A=(1,3,5,6)$ and take $w=(-4,-2,0,1)$ which satisfies the assumption in Theorem 4.3. Then the initial ideal $\operatorname{in}_{(-w, w)}\left(H_{A}(\beta)\right)$ is generated by $\left\{\partial_{2}, \partial_{3}, \partial_{4}\right\}$ and $\theta_{1}+3 \theta_{2}+5 \theta_{3}+6 \theta_{4}-\beta$. The rank of this system is 1 and the solution of this system is spanned by $x_{1}^{\beta}$. We extend this solution to a series solution of $H_{A}(\beta)$. The solution can be written as

$$
\phi_{v}=\sum_{u \in N_{v}} \frac{[v]_{u_{-}}}{[v+u]_{u_{+}}} x^{v+u}=x_{1}^{\beta}\left(1+\frac{\beta(\beta-1)(\beta-2)}{1 !} x_{1}^{-3} x_{2}+\cdots\right),
$$

where $v=(\beta, 0,0,0)$. The corresponding series solution of the modified system is $\psi_{v}(x, t)=\phi_{v}\left(t^{-4} x_{1}, t^{-2} x_{2}, x_{3}, t x_{4}\right)$.

The Gevrey index $r+1=1 / \kappa+1$ of the series $\psi_{v}(x, t)$ with respect to $t=0$ is $1 / 5+1$ if $\beta$ is very generic; see Remark 4.4. Apply the Borel transformation (4.14). The transformed series

$$
x_{1}^{\beta} \zeta^{-4 \beta / 5}\left(\frac{1}{\Gamma\left(1-\frac{4}{5} \beta\right)}+\frac{\beta(\beta-1)(\beta-2)}{1 !} x_{1}^{-3} x_{2} \frac{\zeta^{2}}{\Gamma\left(1-\frac{4}{5} \beta+2\right)}+\cdots\right)
$$

satisfies the $A$-hypergeometric system associated to the matrix

$$
A_{B}=\left(\begin{array}{rrrrr}
1 & 3 & 5 & 6 & 0 \\
-4 & -2 & 0 & 1 & -5
\end{array}\right)
$$

and $\beta_{B}=(\beta, 0)$, which is a regular holonomic system.

Let us introduce the hypergeometric series

$$
\sum_{m, n, k \geq 0,2 k+5 n+2 e_{p} \leq m} \frac{z_{3}^{k} z_{4}^{2 n+e_{p}} z_{5}^{2 m+e_{p}}}{[b]_{k+3 n-3 m}\left(-2 k-5 n+m-2 e_{p}\right) ! k !\left(2 n+e_{p}\right) ![a]_{2 m+e_{p}}}
$$

depending on an integer $e_{p}$. We denote it by $F_{\text {odd }}\left(a, b ; z_{3}, z_{4}, z_{5}\right)$ when $e_{p}=1$ and by $F_{\text {even }}\left(a, b ; z_{3}, z_{4}, z_{5}\right)$ when $e_{p}=0$. Then the series (4.16) is expressed as

$$
\frac{x_{1}^{\beta} \zeta^{-4 \beta / 5}}{\Gamma\left(1-\frac{4}{5} \beta\right)} F\left(x_{1} x_{2}^{-2} x_{3}, x_{1}^{3 / 2} x_{2}^{-5 / 2} x_{4}, x_{1}^{-3 / 2} x_{2}^{1 / 2} \zeta\right)
$$

where

$$
F(z)=F_{\text {odd }}\left(1-\frac{4}{5} \beta, \beta+1 ; z\right)+F_{\text {even }}\left(1-\frac{4}{5} \beta, \beta+1 ; z\right) .
$$

It follows from (4.7) that the series $\psi_{v}(x, t)$ is an asymptotic expansion of

$$
\int_{0}^{e^{i \theta} \infty} e^{-(\tau / t)^{5}} \frac{x_{1}^{\beta} \tau^{-4 \beta / 5}}{\Gamma\left(1-\frac{4}{5} \beta\right)} F\left(x_{1} x_{2}^{-2} x_{3}, x_{1}^{3 / 2} x_{2}^{-5 / 2} x_{4}, x_{1}^{-3 / 2} x_{2}^{1 / 2} \tau\right) \frac{5 \tau^{4}}{t^{5}} d \tau .
$$




\section{BOREL TRANSFORMATION REVISITED}

In this section we assume that the $\mathbb{Q}$-row span of the matrix $A$ does not contain the vector $(1, \ldots, 1)$ and we see that by studying the irregularity of the modified system $\mathcal{M}_{A, w, \alpha}(\beta)$ along $T$ we can give an analytic meaning to the Gevrey series solutions of the $A$-hypergeometric system $\mathcal{M}_{A}(\beta)$ along coordinate varieties constructed in [10. More precisely, we prove (Remark 5.7 and Theorem 5.8) that these Gevrey solutions of $\mathcal{M}_{A}(\beta)$ are asymptotic expansions of certain holomorphic solutions of $\mathcal{M}_{A}(\beta)$ when some conditions are satisfied.

Recall that a vector $v$ is said to be associated with a simplex $\sigma$ if $v=v^{\mathbf{k}}$ is such that $v_{i}=k_{i} \in \mathbb{N}$ for all $i \notin \sigma$ and $A v=\beta$. Let us also recall that $\Delta_{A}$ is the convex hull of the columns of $A$ and the origin while $\operatorname{conv}(A)$ is the convex hull of the columns of $A$.

The Gevrey series solutions constructed in [10 are of the form $\phi_{v}$ for $v$ associated with a suitable simplex $\sigma$ of $A$. In order to interpret some of these Gevrey series as an asymptotic expansion of a solution of $M_{A}(\beta)$ via the modified $A$-hypergeometric system, we consider a vector $w \in \mathbb{Z}^{n}$ with the following coordinates:

$$
w_{i}= \begin{cases}\left|\operatorname{det}\left(A_{\sigma}\right)\right|\left(\left|A_{\sigma}^{-1} a_{i}\right|-1\right) & \text { if }\left|A_{\sigma}^{-1} a_{i}\right|>1, \\ 0 & \text { otherwise }\end{cases}
$$

up to addition with a linear combination of the rows of $A$. Notice that when $\sigma$ is contained in a facet of $\operatorname{conv}(A)$ that is not a facet of $\Delta_{A}$, this vector $w$ verifies the assumptions of Theorem 4.3. More precisely, $\left|\operatorname{det}\left(A_{\sigma}\right)\right|(1, \ldots, 1)+w$ is a linear combination of the rows of $A$.

Remark 5.1. Notice that for $w$ given by (5.1) all the columns $\widetilde{a}_{i}$ of the matrix $\widetilde{A}(w)$ except for $\widetilde{a}_{n+1}$ are contained in the union of at most two hyperplanes. If we take coordinates $\left(y, y_{d+1}\right)$ in $\mathbb{R}^{d} \times \mathbb{R}$, then the hyperplane $\left\{y_{d+1}=0\right\}$ contains all the columns $\tilde{a}_{i}$ such that $\left|A_{\sigma}^{-1} a_{i}\right| \leq 1$ and the hyperplane $\left\{\left|A_{\sigma}^{-1} y\right|-\frac{1}{\left|\operatorname{det}\left(A_{\sigma}\right)\right|} y_{d+1}=\right.$ $1\}$ contains $-\left|\operatorname{det}\left(A_{\sigma}\right)\right| \widetilde{a}_{n+1}$ and all the columns $\widetilde{a}_{i}$ such that $\left|A_{\sigma}^{-1} a_{i}\right| \geq 1$. In particular, the intersection of these two hyperplanes contains all the columns $\widetilde{a}_{i}$ such that $\left|A_{\sigma}^{-1} a_{i}\right|=1$ (for example, all the columns $\widetilde{a}_{i}$ for $i \in \sigma$ ). We also notice that the points $\left\{\widetilde{a}_{i}: i=1, \ldots, n\right\} \cup\left\{-\left|\operatorname{det}\left(A_{\sigma}\right)\right| \widetilde{a}_{n+1}\right\}$ belong to the same hyperplane if and only if $\sigma$ is contained in a facet of $\operatorname{conv}(A)$ that is not a facet of $\Delta_{A}$. Notice that if $\sigma$ is contained in a facet of $\Delta_{A}$ and $v$ is associated with $\sigma$, then the corresponding $w$ given by (5.1) is 0 and $\phi_{v}(x)$ is convergent. This remark proves the following:

Lemma 5.2. If $\sigma$ is a $(d-1)$-simplex of $A$ not contained in a facet of $\Delta_{A}$ and $w$ is given by (5.1), then $s^{\prime}=r^{\prime}+1=1+1 /\left|\operatorname{det}\left(A_{\sigma}\right)\right|$ is a slope of $\mathcal{M}_{A, w, \alpha}(\beta)$ along $T$, for any $\alpha$.

Proposition 5.3. Let $\sigma$ be a $(d-1)$-simplex of $A$ not contained in a facet of $\Delta_{A}$ and consider $w$ given by (5.1) and $\beta$ very generic. For any vector $v$ associated with $\sigma$ we have that, up to multiplication by $t^{\alpha-w v}$, the series $\psi(x, t)=t^{-\alpha} \phi_{v}\left(t^{w} x\right)$ is a Gevrey solution of $\mathcal{M}_{A, w, \alpha}(\beta)$ with index $s^{\prime}=r^{\prime}+1=1+1 /\left|\operatorname{det}\left(A_{\sigma}\right)\right|$ along $T$ at any point of $T \cap U_{\sigma, R}$ for some $R>0$, where $U_{\sigma, R}=\left\{(x, t) \in \mathbb{C}^{n} \times \mathbb{C}:\left|x_{j} t^{w_{j}}\right|<\right.$ $R\left|x_{\sigma}^{A_{\sigma}^{-1} a_{j}}\right|$ if $j \notin \sigma$ and $\left.\left|A_{\sigma}^{-1} a_{j}\right| \geq 1\right\} \cap\left\{x_{i} \neq 0: i \in \sigma\right\}$.

Proof. By [10, Theorem 3.11] we have that $\phi_{v}(x)$ is a Gevrey solution of $\mathcal{M}_{A}(\beta)$ with index $\left.s=r+1=\max _{i}\left\{\left|A_{\sigma}^{-1} a_{i}\right|\right\}\right)$ along $Y=\left\{x_{i}=0:\left|A_{\sigma}^{-1} a_{i}\right|>1\right\}$ at any 
point of $Y \cap\left\{x \in \mathbb{C}^{n}:\left|x_{j}\right|<R\left|x_{\sigma}^{A_{\sigma}^{-1} a_{j}}\right|\right.$ if $j \notin \sigma$ and $\left.\left|A_{\sigma}^{-1} a_{j}\right|=1\right\} \cap\left\{x_{i} \neq 0: i \in \sigma\right\}$, for some $R>0$.

It is clear from (5.1) that $w \in \mathbb{N}^{n}$ and $w_{j}=0$ for all $j \in \sigma$. Hence, for any exponent $v+u$ in the series $\phi_{v}(x)$ the corresponding exponent of $t$ in $\psi(x, t)$ is $-\alpha+w(v+u)=-\alpha+\sum_{j \notin \sigma} w_{j}\left(v_{j}+u_{j}\right) \in-\alpha+\mathbb{N}$ because $v_{j} \in \mathbb{N}$ for all $j \notin \sigma$ and $u \in N_{v}$. We conclude the proof by using Remark 3.21.

In analogy with Section 4 we denote $\psi_{B}(x, \tau)=\hat{\mathcal{B}}_{1 / r^{\prime}}[\psi](x, \tau)$, which defines a holomorphic function at any point in $U_{\sigma, R}$ for some $R>0$ by Proposition 5.3 . We also denote $\varphi(x, z)=\psi\left(x, z^{r^{\prime}}\right)$, and hence $\varphi_{B}(x, \zeta)=\hat{\mathcal{B}}_{1}[\varphi](x, \zeta)=\psi_{B}\left(x, \zeta^{r^{\prime}}\right)$ is convergent at points in the open set

$$
U_{\sigma, R}^{\prime}=\left\{(x, \zeta) \in \mathbb{C}^{n} \times \mathbb{C}: \zeta=\tau^{\left|\operatorname{det}\left(A_{\sigma}\right)\right|},(x, \tau) \in U_{\sigma, R}\right\} .
$$

Moreover, the series $\varphi_{B}(x, \zeta)$ is a holomorphic solution of the hypergeometric system $H_{A_{B}}\left(\beta_{B}\right)$ (in the variables $(x, \zeta)$ ), where

$$
A_{B}=\left(\begin{array}{cc}
A & 0 \\
w & -\kappa
\end{array}\right), \quad \beta_{B}=\left(\begin{array}{c}
\beta \\
\alpha
\end{array}\right) \quad \text { with } \kappa=\left|\operatorname{det}\left(A_{\sigma}\right)\right| .
$$

Lemma 5.4. Assume $\Phi_{A_{\eta}}^{F, d-1} \subseteq \Phi_{A}^{F, d-1}$ where $\eta=\left\{i:\left|A_{\sigma}^{-1} a_{i}\right| \geq 1\right\}$. Let $\widetilde{\sigma} \subseteq$ $\tau \in \Phi_{A_{\eta}}^{F, d-1}$ be a simplex and $\widetilde{v} \in \mathbb{C}^{n+1}$ a vector associated with $\widetilde{\sigma} \cup\{n+1\}$ (i.e. $A_{B} \widetilde{v}=\beta_{B}$ and $\widetilde{v}_{i} \in \mathbb{N}$ for all $\left.i \notin \widetilde{\sigma} \cup\{n+1\}\right)$. The series $\phi_{\widetilde{v}}(x, \zeta)$ converges at points $(x, \zeta) \in U \times\left\{\zeta:|\zeta|>R^{\prime}\right\}$, and for arbitrarily small $c_{2}>0$ we can choose $c_{1}>0$ such that $\left|\phi_{\widetilde{v}}(x, \zeta)\right| \leq c_{1} \exp \left(c_{2}|\zeta|\right)$.

Remark 5.5. The previous condition on the $(A, F)$-umbrella holds for any $d \times n$ matrix $A$ with $d=1$ or $n-1=d$. Moreover, for any $(d, n)$ there are infinitely many matrices $A$ satisfying the condition.

Proof. Notice that $\eta \cup\{n+1\}$ is a facet of the $\left(A_{B}, F\right)$-umbrella and $\widetilde{\sigma} \cup\{n+1\}$ is a simplex of $A_{B}$ contained in $\eta \cup\{n+1\}$. In particular, we know that $\phi_{\widetilde{v}}$ is convergent in a certain open set. Let us denote $\kappa=\left|\operatorname{det}\left(A_{\sigma}\right)\right|$. If $\left\{b_{i}: i \notin \widetilde{\sigma} \cup\{n+1\}\right\}$ is the basis of $\operatorname{ker}\left(A_{B}\right)$ associated with $\widetilde{\sigma} \cup\{n+1\}$, then the coordinate sum of $b_{i}$ is

$$
\left|b_{i}\right|=\left\{\begin{array}{cc}
0 & \text { if } i \in \eta \backslash \widetilde{\sigma} \\
-\left|A_{\widetilde{\sigma}}^{-1} a_{i}\right|+1-\frac{1}{\kappa} w_{\widetilde{\sigma}} A_{\widetilde{\sigma}}^{-1} a_{i} & \text { if } i \notin \eta
\end{array}\right.
$$

Let us denote by $\left(b_{i}\right)_{A}$ the vector given by the first $n$ entries of $b_{i}$. Since $\widetilde{\sigma} \cup$ $\{n+1\} \subseteq \eta \cup\{n+1\} \in \Phi_{A_{B}}^{F, d-1}$, we have that $\left|b_{i}\right|>0$ for all $i \notin \eta$ and that the series $\phi_{\widetilde{v}}$ defines a multivalued holomorphic function in the open set $\{(x, \zeta)$ : $\left.\left|x^{\left(b_{i}\right)_{A}} \zeta^{\left(b_{i}\right)_{n+1}}\right|<R, i \in \eta \backslash \widetilde{\sigma}\right\} \cap\left\{x_{j} \neq 0: j \in \widetilde{\sigma}\right\}$ for some $R>0$. The fact that $\tilde{\sigma} \subseteq \tau \in \Phi_{A_{\eta}}^{F, d-1} \subseteq \Phi_{A}^{F, d-1}$ guarantees that $-\left|A_{\tilde{\sigma}}^{-1} a_{i}\right|+1 \geq 0$ for all $i \in \eta$ and $-\left|A_{\widetilde{\sigma}}^{-1} a_{i}\right|+1>0$ for all $i \notin \eta$. Thus, if $i \in \eta \backslash \widetilde{\sigma}$ the last coordinate of $b_{i}$ is $\left(b_{i}\right)_{n+1}=\left|b_{i}\right|-\left|\left(b_{i}\right)_{A}\right|=-1+\left|A_{\widetilde{\sigma}}^{-1} a_{i}\right| \leq 0$, while if $i \notin \eta$ the last coordinate of $b_{i}$ can be positive.

However, if $\left(b_{i}\right)_{n+1}>0$ for some $i \notin \eta$, we still have that $\left|b_{i}\right|>\left(b_{i}\right)_{n+1}=$ $-\frac{1}{\kappa} w_{\widetilde{\sigma}} A_{\widetilde{\sigma}}^{-1} a_{i}$. In this case there exist $K_{1}, K_{2}>0$ such that

$$
\sum_{m \geq 0}(m !)^{-\left|b_{i}\right|}\left|x^{\left(b_{i}\right)_{A}} \zeta^{\left(b_{i}\right)_{n+1}}\right|^{m} \leq K_{1} \exp \left(K_{2}\left|x^{\left(b_{i}\right)_{A}}\right|^{1 /\left|b_{i}\right|}|\zeta|^{\left(b_{i}\right)_{n+1} /\left|b_{i}\right|}\right),
$$

where $\left(b_{i}\right)_{n+1} /\left|b_{i}\right|<1$. 
On the other hand, if $\widetilde{v}_{i}=k_{i}$ for $i \notin \widetilde{\sigma} \cup\{n+1\}$, it can be shown by using standard estimates on $\Gamma$-functions (see e.g. [14, Proposition 1, Section 1.1], [25, Lemma 1] and [10, Lemma 3.8]) that there exists $C_{1}, C_{2}>0$ such that

$$
\left|\phi_{\widetilde{v}}(x, \zeta)\right| \leq C_{1}\left|x_{\widetilde{\sigma}}^{A_{\widetilde{\sigma}}^{-1} \beta} \zeta^{\frac{-\alpha+w_{\tilde{\sigma}} A_{\tilde{\sigma}}^{-1} \beta}{\kappa}}\right| \prod_{i \notin \widetilde{\sigma} \cup\{n+1\}}\left(\sum_{k_{i}+m_{i} \in \mathbb{N}} \frac{\left(C_{2} \mid x^{\left.\left(b_{i}\right)_{A} \zeta^{\left(b_{i}\right)_{n+1}} \mid\right)^{\left(k_{i}+m_{i}\right)}}\right.}{\left(k_{i}+m_{i}\right) ! b_{i} \mid}\right),
$$

and for all $i \notin \widetilde{\sigma} \cup\{n+1\}$ we have that the previous expression in brackets is bounded above by

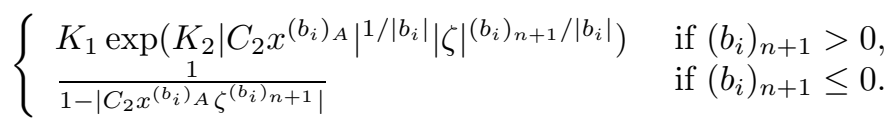

Take $U=\left\{x \in \mathbb{C}^{n}:\left|x^{\left(b_{i}\right)_{A}}\right|<R_{i}, i=1, \ldots, n\right\}$, where $R_{i}>0$ can be chosen arbitrarily large except when $\left(b_{i}\right)_{n+1}=\left|\left(b_{i}\right)_{A}\right|=0$, in which case we take $R_{i}<$ $1 / C_{2}$. Then, since $\left(b_{i}\right)_{n+1} /\left|b_{i}\right|<1$ if $\left(b_{i}\right)_{n+1}>0$, we have the result for arbitrarily small $c_{2}>0$ if we take $c_{2}>0$ and $R^{\prime}>0$ big enough.

Proposition 5.6. $\varphi_{B}(x, \zeta)$ has an analytic continuation to an open set of the form $U \times S(\theta, \delta)$, where $U$ is certain open set of $\mathbb{C}^{n}$ and $S(\theta, \delta)$ is a sector with bisecting direction $\theta$ and a small enough opening $\delta>0$. Moreover, if $\Phi_{A_{\eta}}^{F, d-1} \subseteq \Phi_{A}^{F, d-1}$, then for arbitrarily small $c_{2}>0$ we can chose $c_{1}>0$ such that $\left|\varphi_{B}(x, \zeta)\right| \leq c_{1} \exp \left(c_{2}|\zeta|\right)$ for $(x, \zeta) \in U \times S(\theta, \delta)$.

Proof. To simplify the exposition we will first assume that $\alpha$ is very generic. Notice that $\eta \cup\{n+1\}$ is the set of (indices of) columns of $A_{B}$ belonging to the hyperplane $H=\left\{\left|A_{\sigma}^{-1} y\right|-\frac{1}{\left|\operatorname{det}\left(A_{\sigma}\right)\right|} y_{d+1}=1\right\}$ (see Remark [5.1) and we denote by $A^{\prime}$ the submatrix of $A_{B}$ consisting of these columns. Let $q$ be the cardinality of $\eta$. Recall that $\varphi_{B}(x, \zeta)$ defines a holomorphic function at each point of $U_{\sigma, R}^{\prime}$ (see Proposition 5.3 and (5.2) ) and notice that $w_{j}=0$ for all $j \notin \eta$.

We can write

$$
\varphi_{B}(x, \zeta)=\sum_{m \in \mathbb{N}^{n}-q} \varphi_{m} \frac{x_{\bar{\eta}}^{m}}{m !},
$$

where $\varphi_{m}=\varphi_{m}\left(x_{\eta}, \zeta\right)$ is a holomorphic solution of the hypergeometric system $H_{A^{\prime}}\left(\beta-\sum_{i \notin \eta} m_{i} a_{i}, \alpha\right)$, which is regular holonomic because all the columns of $A^{\prime}$ belong to the hyperplane $H\left[18\right.$. Let $W \subset \mathbb{C}^{q+1}$ be the open set such that $U_{\sigma, R}^{\prime}=$ $W \times \mathbb{C}^{n-q}$ (see (5.2) $)$, so that for all $m=\left(m_{i}\right)_{i \notin \eta} \in \mathbb{N}^{n-q}, \varphi_{m}$ is holomorphic in $W$. Take $Z=\left\{x_{i}=0: i \notin \eta\right\}$ and notice that we can identify $W$ with a relative open subset of $Z$, i.e. with $W \times\{0\}=U_{\sigma, R}^{\prime} \cap Z$.

Recall that the singular locus of a hypergeometric system does not depend on the parameter but only on the matrix (see [1] and [14]). In particular, since $\varphi_{m}$ is convergent in $W$ for all $m$, we can consider the analytic continuation of all the $\varphi_{m}$ along the same path starting at a point in $W$ and avoiding the singular locus of the hypergeometric system associated with $A^{\prime}$. Let $c=\left(c_{i}\right)_{i \in \eta} \in \mathbb{C}^{q}$ be such that the complex line $\left\{x_{i}=c_{i}: i \in \eta\right\} \cap Z$ (with coordinate $\zeta$ ) intersects $W$ at nonsingular points of $H_{A^{\prime}}(\beta, \alpha)$. Notice that this intersection is a relative open set in the complex line. Let $\operatorname{Sing}(c)$ be the set of points $\zeta_{0} \in \mathbb{C} \backslash\{0\}$ such that $\left(x_{\eta}, \zeta\right)=\left(c, \zeta_{0}\right)$ is a singular point of $H_{A^{\prime}}\left(\beta-\sum_{i \notin \eta} m_{i} a_{i}, \alpha\right)$. $\operatorname{Sing}(c)$ is a finite set and thus $\Theta(c)=\{\arg (u): u \in \operatorname{Sing}(c)\}$ is also finite. As we vary $c$ in a small open set $W^{\prime} \subseteq \mathbb{C}^{q}, \Theta(c)$ is contained in a finite union of small intervals and we 
can take $\theta$ such that for $\delta>0$ small enough, $(\theta-\delta / 2, \theta+\delta / 2) \cap \Theta(x)=\emptyset$ for all $x \in W^{\prime}$. Hence we can consider the analytic continuation of each $\varphi_{m}$ to an open set containing $W^{\prime} \times S(\theta, \delta)$.

We have extended $\varphi_{B}$ as a formal solution of $M_{A_{B}}(\beta, \alpha)$ along $Z$, which is convergent in some relative open set of $Z$. Thus, by the constructibility of the solutions of a holonomic system in the sheaf $\mathcal{O}_{\widehat{X \mid Z}} / \mathcal{O}_{X \mid Z}$ (see 24]) we have that the formal solution constructed is convergent at any point of $W^{\prime} \times S(\theta, \delta) \times\{\underline{0}\}$; therefore $\varphi_{B}(x, \zeta)$ can be analytically continued to an open set containing $W^{\prime} \times S(\theta, \delta) \times\{\underline{0}\}$.

Let us see that the analytic continuation of $\varphi_{B}(x, \zeta)$ satisfies a growth estimate near $\zeta=\infty$. Each $\varphi_{m}$ has polynomial growth since it is a solution of the regular hypergeometric system $H_{A^{\prime}}\left(\beta-\sum_{i \notin \eta} m_{i} a_{i}, \alpha\right)$. Since $\alpha, \beta$ are very generic, each $\varphi_{m}$ can be written as a Nilsson series that converges in a certain open set (see e.g. [26, Proposition 3.4.4]) which is a linear combination of series of the form $\phi_{v(m)}\left(x_{\eta}, \zeta\right)$ (for some set of exponents $v(m)$ associated with simplices in a certain regular triangulation of the matrix $\left.A^{\prime}\right)$ with support $N_{v(m)}$ given by integer vectors in $\operatorname{ker}\left(A^{\prime}\right)$ with coordinates sum equal to zero. The open set where the Nilsson series converge depends on the regular triangulation of $A^{\prime}$ that the simplices belong to. We need to use a Nilsson series expression of $\varphi_{m}$ that converges in points $\left(x_{\eta}, \zeta\right)=(c, \zeta)$ with $|\zeta|>R$ for a sufficiently large $R>0$. It is enough to consider a regular triangulation $T$ of $A_{\eta}$ and take $T^{\prime}=\{\widetilde{\sigma} \cup\{n+1\}: \widetilde{\sigma} \in T\}$ as the regular triangulation of $A^{\prime}$. By properties of regular triangulations, there is one regular triangulation $T$ of $A_{\eta}$ such that there exists $c$ as above so that if $|\zeta|>R$ for a sufficiently large $R>0$, then $(c, \zeta)$ belongs to the domain of convergence of the series $\phi_{\widetilde{v}}$ for any vector $\widetilde{v}$ associated with $\widetilde{\sigma} \cup\{n+1\} \in T^{\prime}$.

The series expression of $\varphi_{B}$ via substitution of each $\varphi_{m}$ by its Nilsson series expansion is a formal Nilsson series (see e.g. [10, Lemma 6.15]). We know that this Nilsson series converges to $\varphi_{B}$ at points in $W^{\prime} \times S(\theta, \delta) \times\{\underline{0}\}$ close to $\zeta=\infty$. By Lemma 5.4 it verifies the desired growth estimate.

Notice that the Nilsson series expansion of $\varphi_{B}$ near $\zeta=\infty$ also provides an analytic continuation to points with $|\zeta|$ big enough and $x$ varying in a certain open set of $\mathbb{C}^{n}$ that contains $(c, 0)$.

We have considered analytic continuations along paths contained in $Z$. We can also extend $\varphi_{B}$ by analytic continuation along paths from a point in $W \times \mathbb{C}^{n-q}$ to a point near $\zeta=\infty$ avoiding the singular locus of $M_{A_{B}}(\beta, \alpha)$. If the starting point of the path is close to $(c, 0)$, the analytic continuations coincide with the Nilsson series close to $\zeta=\infty$ and thus it satisfies the same growth estimate.

Finally, the parameter $\beta$ being very generic, the rank of $M_{A_{B}}\left(\beta_{B}\right)$ equals $\operatorname{vol}\left(A_{B}\right)$ since the set of exceptional parameters has codimension at least 2 [23, Porism 9.5]. So, we can reduce the general case (when $\alpha$ is not necessarily generic) to the previous one.

Remark 5.7. Using Proposition 5.6, the results in Section 4 also hold for $w$ as in (5.1) instead of $w$ satisfying the assumption in Theorem 4.3 if we assume the additional condition $\Phi_{A_{\eta}}^{F, d-1} \subseteq \Phi_{A}^{F, d-1}$ to hold. In particular, we obtain an analogous version of Corollary 4.9. With the notation in Proposition 5.3 (we put $\alpha=0$ to simplify expressions), let $\mathcal{S}[\psi](x, t)$ be the $\kappa$-sum of $\psi(x, t)$ with respect to $t$ in a direction $\theta \notin \Theta(x)$ for $x$ in a certain open set $U$ small enough with compact closure. For any closed subsector $\bar{S}$ of $S(\theta, \varpi, \rho)$ (see the notation in Section 4 ) there exist 
constants $C>0$ and $K>0$ such that the inequality $\left|\mathcal{S}[\psi](c, t)-\psi_{N}(c, t)\right| \leq$ $C K^{N} \Gamma(1+N / \kappa)|t|^{N}$ holds for $t \in \bar{S}, c=\left(c_{1}, \ldots, c_{n}\right) \in U$ and any $N \in \mathbb{N}$. Thus, considering the parametric curve $x(t)=\left(c_{1} t^{w_{1}}, \ldots, c_{n} t^{w_{n}}\right)$, then $\psi_{N}(x(t), 1)=$ $\psi_{N}(c, t)$ and $\mathcal{S}[\psi](x(t), 1)=\mathcal{S}[\psi](c, t)$. Notice that $x$ tends to the point $x^{\prime} \in Y$, with $x_{i}^{\prime}=x_{i}$ if $\left|A_{\sigma}^{-1} a_{i}\right| \leq 1$, as $t$ tends to 0 .

Theorem 5.8. Let $\beta \in \mathbb{C}^{n}$ be very generic and let $\phi_{v}$ be a Gevrey solution of $H_{A}(\beta)$ of order $s=1+1 / k>1$ with respect to a coordinate hyperplane $Y=\left\{x_{i}=0\right\}$. Let $\sigma$ be the simplex which $v$ is associated with and take $w$ associated with $\sigma$ as well. If $\Phi_{A_{\eta}}^{F, d-1} \subseteq \Phi_{A}^{F, d-1}$, then for $\kappa=\left|\operatorname{det}\left(A_{\sigma}\right)\right|$ we have that $\mathcal{S}[\psi](x, 1)$ is a holomorphic solution of $M_{A}(\beta)$ and that for each $\left(x_{1}, \ldots, x_{i-1}, x_{i+1}, \ldots, x_{n}\right)$ in a certain open set of $\mathbb{C}^{n-1}, \phi_{v}(x)$ is a Gevrey asymptotic expansion of order $s$ of $\mathcal{S}[\psi](x, 1)$ with respect to $x_{i}=0$ in all but finitely many directions.

Proof. Assume for simplicity that the hyperplane is $Y=\left\{x_{n}=0\right\}$, and so $\sigma \subseteq$ $\{1, \ldots, n-1\}$. We have that $w_{i}=0$ for $i=1, \ldots, n-1$ and $w_{n}=\left|\operatorname{det}\left(A_{\sigma}\right)\right|(s-1)>$ 0 , where $s=\left|A_{\sigma}^{-1} a_{n}\right|>1$ is the Gevrey index of $\phi_{v}$ along $Y$. By Remark 5.7 for $x(t)=\left(c_{1}, \ldots, c_{n-1}, c_{n} t^{w_{n}}\right)$ with $t \in \bar{S}$ and $c \in U$, we have the inequality

$$
\left|\mathcal{S}[\psi](x(t), 1)-\psi_{N}(x(t), 1)\right| \leq C K^{N} \Gamma(1+N / \kappa)|t|^{N}
$$

for all $N \geq 0$. Here we write $\psi_{N}(x, 1)=\sum_{w_{n} m<N} f_{m}\left(x_{1}, \ldots, x_{n-1}\right) x_{n}^{m}$. The result is proven by taking $N=w_{n} M$ with $M \in \mathbb{N}$.

Example 5.9. This example shows that the hypothesis in Proposition 5.6 on the umbrella is necessary and that the bound there is sharp. Take

$$
A=\left(\begin{array}{llll}
1 & 1 & 0 & \ell \\
0 & 1 & 2 & 0
\end{array}\right)
$$

where $\ell>1$ is a rational number. We can consider $\ell \in \mathbb{Q}$ by changing the lattice $\mathbb{Z}^{2}$ by the lattice $\left(\frac{1}{\ell} \mathbb{Z}\right) \times \mathbb{Z}$. Then the weight vector $w=(0,0,0, \ell-1)$ is associated with $\sigma=\{1,2\}$ by the formula (5.1) and $\kappa=\left|\operatorname{det} A_{\sigma}\right|=1$. Let $\alpha \in \mathbb{C}$ and $\beta \in \mathbb{C}^{2}$ be very generic and let $v \in \mathbb{C}^{4}$ be a vector associated with $\sigma$ so that the series $\phi_{v}(x)$ is a Gevrey solution of $M_{A}(\beta)$ along $x_{4}=0$ with Gevrey index $s=1+r=\ell>1$. Then for $\psi_{v}(x, t)=t^{-\alpha} \phi_{v}\left(x_{1}, x_{2}, x_{3}, t^{\ell-1} x_{4}\right)$ the Borel transform $\varphi_{B}(x, \zeta)$ is convergent in the open set $\left\{(x, \zeta):\left|\zeta^{\ell-1}\right|<\epsilon\left|x_{1}^{\ell} / x_{4}\right|\right\}$ for some $\epsilon>0$ small enough.

Moreover, it defines a holomorphic solution of $M_{A_{B}}\left(\beta_{B}\right)$ and then it is a linear combination of the set of series $\phi_{v^{\prime}}(x, \zeta)$ with $v^{\prime} \in \mathbb{C}^{5}$ associated with the simplex $\{1,2,5\}$. Its analytic continuation to points in the open set $\left\{(x, \zeta):\left|\zeta^{\ell-1}\right|>\right.$ $\left.R\left|x_{1}^{\ell} / x_{4}\right|\right\}$ for some sufficiently large $R>0$ is a linear combination of the set of series $\phi_{\widetilde{v}}$ with $\widetilde{v} \in \mathbb{C}^{5}$ associated with the simplex $\{2,4,5\}$ of $A_{B}$. The column vectors of $B_{\{2,4,5\}}$ are $b_{1}=(1,0,0,-1 / \ell,(1-\ell) / \ell)$ and $b_{3}=(0,-2,1,2 / \ell, 2(\ell-1) / \ell)$. Elements in the support $N_{\widetilde{v}}$ of $\phi_{\widetilde{v}}$ are of the form $m_{1} b_{1}+m_{3} b_{3} \in \mathbb{Z}^{5}$ with $m_{1}, m_{3} \in \mathbb{N}$. Thus any of these series $\phi_{\widetilde{v}}(x, \zeta)$ is convergent in the open set $\left\{(x, \zeta):\left|\zeta^{\ell-1}\right|>\right.$ $\left.R\left|x_{1}^{\ell} / x_{4}\right|\right\}$ for some sufficiently large $R>0$, and since the last coordinate of $b_{3}$ is $2(\ell-1) / \ell>0$ there is a subseries of $\phi_{\widetilde{v}}$ (the one with monomials $(x, \zeta)^{\widetilde{v}+m_{3} b_{3}}$, $\left.2 m_{3}(\ell-1) / \ell \in \mathbb{N}\right)$ such that the set of exponents of $\zeta$ in its monomials is contained in $\widetilde{v}_{5}+\mathbb{N}$. The fact that $\left|b_{3}\right|=1>0$ guarantees that the coefficients of this subseries has the same type of growth as $1 /\left(m_{1}\right)$ !, and thus the growth of this series, as $\zeta$ tends to $\infty$ in a certain sector, is equivalent to the growth of $K \exp \left(C x_{3} x_{4}^{2 / \ell} \zeta^{2(\ell-1) / \ell} / x_{2}^{2}\right)$ for some $K, C>0$. Notice that for $\ell>1$, we have that $\eta=\{1,2,4\}$ and the 
hypothesis $\Phi_{A_{\eta}}^{F, d-1} \subseteq \Phi_{A}^{F, d-1}$ (required in Lemma 5.4 and Proposition 5.6) is satisfied if and only if $1<\ell<2$. Thus, the bound $\left|\phi_{\widetilde{v}}(x, \zeta)\right| \leq c_{1} \exp \left(c_{2}|\zeta|\right)$ is satisfied for some $c_{1}, c_{2}>0$ if and only if $1<\ell \leq 2$, but for $\ell=2$ we cannot choose $c_{2}$ to be arbitrarily small.

\section{ACKNOWLEDGEMENTS}

We wish to thank J. González-Meneses, M. Granger and D. Mond for their help, suggestions and comments. We are very grateful to an anonymous referee, whose thoughtful suggestions have improved this article.

\section{REFERENCES}

[1] Alan Adolphson, Hypergeometric functions and rings generated by monomials, Duke Math. J. 73 (1994), no. 2, 269-290, DOI 10.1215/S0012-7094-94-07313-4. MR1262208 (96c:33020)

[2] A. Assi, F. J. Castro-Jiménez, and J. M. Granger, How to calculate the slopes of a D-module, Compositio Math. 104 (1996), no. 2, 107-123. MR.1421395 (98i:32010)

[3] Werner Balser, From divergent power series to analytic functions, Lecture Notes in Mathematics, vol. 1582, Springer-Verlag, Berlin, 1994. Theory and application of multisummable power series. MR.1317343 (96d:34071)

[4] C. Berkesch, J. Forsgård, and M.Passare, Euler-Mellin integrals and A-hypergeometric functions, Michigan Math. J. 63 (2014), no. 1, 101-123. MR3189470

[5] I. N. Bernšter̆n, Modules over a ring of differential operators. An investigation of the fundamental solutions of equations with constant coefficients (Russian), Funkcional. Anal. i Priložen. 5 (1971), no. 2, 1-16. MR0290097 (44 \#7282)

[6] F. Beukers, Monodromy of A-hypergeometric functions, arxiv:1101.0493v2.

[7] Francisco Jesús Castro-Jiménez and Nobuki Takayama, Singularities of the hypergeometric system associated with a monomial curve, Trans. Amer. Math. Soc. 355 (2003), no. 9, 37613775 (electronic), DOI 10.1090/S0002-9947-03-03300-2. MR.1990172 (2004j:32009)

[8] Alicia Dickenstein, Federico Martínez, and Laura Felicia Matusevich, Nilsson solutions for irregular A-hypergeometric systems, Rev. Mat. Iberoam. 28 (2012), no. 3, 723-758, DOI 10.4171/RMI/689. MR2949617

[9] A. Esterov, K. Takeuchi, Confluent A-hypergeometric functions and rapid decay homology cycles, arxiv:1107.0402, 2011.

[10] M. C. Fernández-Fernández, Irregular hypergeometric D-modules, Adv. Math. 224 (2010), no. 5, 1735-1764, DOI 10.1016/j.aim.2010.01.017. MR.2646108 (2011g:13055)

[11] M. C. Fernández-Fernández and F. J. Castro-Jiménez, Gevrey solutions of irregular hypergeometric systems in two variables, J. Algebra 339 (2011), 320-335, DOI 10.1016/j.jalgebra.2011.02.045. MR.2811324

[12] M. C. Fernández-Fernández and F. J. Castro-Jiménez, Gevrey solutions of the irregular hypergeometric system associated with an affine monomial curve, Trans. Amer. Math. Soc. 363 (2011), no. 2, 923-948, DOI 10.1090/S0002-9947-2010-05115-3. MR.2728590 (2011m:32011)

[13] I. M. Gel'fand, M. I. Graev and A. V. Zelevinsky, Holonomic systems of equations and series of hypergeometric type, Dokl. Akad. Nauk SSSR 295 (1987), no. 1, 14-19. MR0902936 (88j:58118)

[14] I. M. Gel'fand, A. V. Zelevinskiǔ, and M. M. Kapranov, Hypergeometric functions and toric varieties (Russian), Funktsional. Anal. i Prilozhen. 23 (1989), no. 2, 12-26, DOI 10.1007/BF01078777; English transl., Funct. Anal. Appl. 23 (1989), no. 2, 94-106. MR,1011353(90m:22025)

[15] I. M. Gel'fand, M. M. Kapranov, and A. V. Zelevinsky, Generalized Euler integrals and A-hypergeometric functions, Adv. Math. 84 (1990), no. 2, 255-271, DOI 10.1016/00018708(90)90048-R. MR1080980 (92e:33015)

[16] María Isabel Hartillo Hermoso, Slopes of hypergeometric systems of codimension one, Proceedings of the International Conference on Algebraic Geometry and Singularities (Spanish) (Sevilla, 2001), Rev. Mat. Iberoamericana 19 (2003), no. 2, 455-466, DOI 10.4171/RMI/357. MR2023195 (2005d:16039) 
[17] María Isabel Hartillo-Hermoso, Irregular hypergeometric systems associated with a singular monomial curve, Trans. Amer. Math. Soc. 357 (2005), no. 11, 4633-4646 (electronic), DOI 10.1090/S0002-9947-04-03614-1. MR2156724 (2006h:32005)

[18] R. Hotta, Equivariant D-modules, arxiv:math/9805021, 1998.

[19] Jan Hrabowski, Multiple hypergeometric functions and simple Lie groups SL and Sp, SIAM J. Math. Anal. 16 (1985), no. 4, 876-886, DOI 10.1137/0516066. MR.793929 (87e:33016)

[20] G. Laumon, Transformations canoniques et spécialisation pour les $\mathcal{D}$-modules filtrés (French), Astérisque 130 (1985), 56-129. Differential systems and singularities (Luminy, 1983). MR 804050 (87h:32027)

[21] Yves Laurent, Polygône de Newton et b-fonctions pour les modules microdifférentiels (French), Ann. Sci. École Norm. Sup. (4) 20 (1987), no. 3, 391-441. MR925721 (89k:58282)

[22] Yves Laurent and Zoghman Mebkhout, Pentes algébriques et pentes analytiques d'un $\mathcal{D}$ module (French, with English and French summaries), Ann. Sci. École Norm. Sup. (4) 32 (1999), no. 1, 39-69, DOI 10.1016/S0012-9593(99)80008-1. MR1670595 (2001b:32015)

[23] Laura Felicia Matusevich, Ezra Miller, and Uli Walther, Homological methods for hypergeometric families, J. Amer. Math. Soc. 18 (2005), no. 4, 919-941 (electronic), DOI 10.1090/S0894-0347-05-00488-1. MR2163866 (2007d:13027)

[24] Zoghman Mebkhout, Le théorème de positivité de l'irrégularité pour les $\mathcal{D}_{X}$-modules (French), The Grothendieck Festschrift, Vol. III, Progr. Math., vol. 88, Birkhäuser Boston, Boston, MA, 1990, pp. 83-132, DOI 10.1007/978-0-8176-4576-2_4. MR1106912 (92j:32031)

[25] Katsuyoshi Ohara and Nobuki Takayama, Holonomic rank of $\mathcal{A}$-hypergeometric differentialdifference equations, J. Pure Appl. Algebra 213 (2009), no. 8, 1536-1544, DOI 10.1016/j.jpaa.2008.11.018. MR2517990 (2010g:16039)

[26] Mutsumi Saito, Bernd Sturmfels, and Nobuki Takayama, Gröbner deformations of hypergeometric differential equations, Algorithms and Computation in Mathematics, vol. 6, SpringerVerlag, Berlin, 2000. MR1734566 (2001i:13036)

[27] Mathias Schulze and Uli Walther, Irregularity of hypergeometric systems via slopes along coordinate subspaces, Duke Math. J. 142 (2008), no. 3, 465-509, DOI 10.1215/001270942008-011. MR2412045 (2009b:13067)

[28] Mathias Schulze and Uli Walther, Irregularity of hypergeometric systems via slopes along coordinate subspaces, arXiv:math/0608668v3 [math.AG].

[29] Bernd Sturmfels, Gröbner bases and convex polytopes, University Lecture Series, vol. 8, American Mathematical Society, Providence, RI, 1996. MR.1363949 (97b:13034)

[30] Nobuki Takayama, Modified $\mathcal{A}$-hypergeometric systems, Kyushu J. Math. 63 (2009), no. 1, 113-122, DOI 10.2206/kyushujm.63.113. MR2522925 (2010m:33020)

Department of Algebra, University of Sevilla, Seville, Spain

E-mail address: castro@us.es

Department of Algebra, University of Sevilla, Seville, Spain

E-mail address: mcferfer@us.es

Department of Mathematics, University of Kobe, Kobe, Japan

E-mail address: koike@math.kobe-u.ac.jp

Department of Mathematics, University of Kobe, Kobe, Japan

E-mail address: takayama@math.kobe-u.ac.jp 